COMPUTATIONAL THERMAL SCIENCES: AN INTERNATIONAL JOURNAL

Accepted February $1^{\text {st }} 2020$

\title{
COMPUTATION OF TRANSIENT RADIATIVE REACTIVE THERMO-SOLUTAL MAGNETOHYDRODYNAMIC CONVECTION IN INCLINED MHD HALL GENERATOR FLOW WITH DISSIPATION AND CROSS DIFFUSION
}

\author{
Siva Reddy Sheri' ${ }^{1}$ O. Anwar Bég ${ }^{2 *}$, Prasanthi Modugula1 ${ }^{1}$ and A. Kadir ${ }^{2 *}$ \\ ${ }^{1}$ Department of Mathematics, GITAM University, Hyderabad Campus, Telangana, India. \\ ${ }^{2}$ Multi-Physical Engineering Sciences Group, Department of Aeronautical and Mechanical Engineering, \\ Newton Building, Salford University, Manchester, M54WT, UK.
}

\begin{abstract}
:
The present article investigates the collective influence of chemical reaction, viscous dissipation and Hall current magnetic effects on timedependent radiative magnetohydrodynamic flow, heat and mass transfer from an inclined wall embedded in a homogenous, isotropic highpermeability porous medium. The model developed is relevant to near wall magnetohydrodynamic energy generator transport phenomena in which chemical corrosion effects may arise during operations. The governing non-linear partial differential equations for mass, momentum, energy and species conservation are transformed into a system of coupled non-linear dimensionless partial differential equations with appropriate similarity variables. The normalized conservation equations are then solved with a robust finite element method (MATLABFEM) subject to corresponding initial and boundary conditions. Important dimensionless parameters emerging are Eckert number, thermal Grashof number, solutal Grashof number, magnetic body force parameter, Hall parameter, permeability parameter, Dufour number, Soret number, time, radiation-conduction parameter, chemical reaction parameter, heat absorption parameter, Prandtl number, Schmidt number and wall angle. Primary velocity is enhanced with Eckert number, thermal Grashof number, solutal Grashof number, Hall parameter, permeability parameter, Dufour number, Soret number, radiation-conduction parameter and time whereas it is reduced with first order chemical reaction parameter, heat absorption, magnetic body force parameter, Prandtl number, Schmidt number and wall inclination. Secondary velocity is elevated with Eckert number, solutal Grashof number, thermal Grashof number, magnetic body force parameter, Hall parameter, radiation-conduction parameter, Dufour number, Soret number and time whereas it is suppressed with reaction parameter, heat absorption, Prandtl number, Schmidt number and wall inclination. Temperature is enhanced with Eckert number, Dufour number, heat absorption, radiation-conduction parameter and time whereas it is depressed with Prandtl number. Species concentration is reduced with increasing chemical reaction parameter (destructive homogenous reaction) and Schmidt number whereas it is elevated with Soret number and time. Extensive discussion of the finite element formulation, convergence and validation is provided Skin friction, Nusselt number and Sherwood number distributions are also provided for selected parameter variation. Validation of solutions with published literature is also included for several special cases, namely non-reactive, non-dissipative flow in the absence of heat generation or absorption. Further validation is included using a multi-step differential transform method (MS-DTM). The present simulations provide an interesting insight into complex fluid/thermal/species diffusion characteristics in the boundary layer region of relevance to working MHD generator systems.
\end{abstract}

Keywords: MHD Hall energy generators; Corrosive chemical reaction, Viscous dissipation, Hall current; thermosolutal buoyancy; Inclination; porous media; heat transfer; cross diffusion; MATLAB-FEM; MS-DTM.

\section{NOMENCLATURE}

$B_{o} \quad$ Magnetic field strength

$C_{p} \quad$ Specific heat at constant pressure

$C_{s} \quad$ Concentration susceptibility

C Dimensionless concentration

$C^{\prime} \quad$ Dimensional concentration

$C_{\infty}^{\prime} \quad$ Concentration in free stream (edge of boundary layer)

$D_{m} \quad$ Chemical molecular diffusivity

Du Dufour (thermo-diffusion) number

Ec Eckert number

$g \quad$ Acceleration due to gravity 
Gr Grashof number for heat transfer (thermal)

Gm Grashof number for mass transfer (solutal)

$K^{\prime} \quad$ Permeability of the porous medium

$k \quad$ Thermal conductivity of the MHD generator working fluid

Kr Dimensionless chemical reaction

$K_{r}^{\prime} \quad$ Rate of chemical reaction

K Permeability parameter (hydraulic conductivity of MHD generator porous medium)

$K_{T} \quad$ Thermal diffusion ratio

m Hall parameter

M Magnetic body force parameter

$\mathrm{Nu} \quad$ Nusselt number

Pr Prandtl number

$q_{r} \quad$ Radiative heat flux in $y$-direction

Q Heat absorption parameter

$R \quad$ Radiation-conduction parameter

Sc Schmidt number

Sh Sherwood number

Sr Soret (diffuso-thermal) number

$T_{m} \quad$ Mean fluid temperature

$t \quad$ Non-dimensional time

$T^{\prime} \quad$ Dimensional temperature

$T_{\infty}^{\prime} \quad$ Temperature of free stream

$u \quad$ Non-dimensional velocity

$u^{\prime} \quad$ Velocity component along $x^{\prime}$-direction

$v^{\prime} \quad$ Velocity component along $y^{\prime}$-direction

$w^{\prime} \quad$ Velocity component along $z^{\prime}$-direction

$x \quad$ Non-dimensional distance along $x$-axis

y Non-dimensional distance along y-axis

$z \quad \quad \quad$ Non-dimensional distance along $z$-axis

Greek 
Inclination angle of MHD generator wall

$\theta$

Dimensionless temperature

$\gamma \quad$ Volumetric coefficient of thermal expansion

$\sigma \quad$ Electrical conductivity of MHD generator working fluid

$\gamma^{*} \quad$ Coefficient of volume expansion for mass transfer

$v \quad$ Kinematic viscosity of MHD generator working fluid

$\mu \quad$ Dynamic viscosity of MHD generator working fluid

$\rho \quad$ Density of MHD generator working fluid

$\tau \quad$ Skin friction coefficient

\section{Subscripts}
w Condition at the wall
$\infty \quad$ Free stream conditions

\section{Superscript}

Denotes quantity in dimensional form

\section{INTRODUCTION}

MHD (magnetohydrodynamic) generators are an important contribution to the global upsurge in renewable energy systems in the $21^{\text {st }}$ century. These exploit magnetohydrodynamics to generate sustainable, clean, durable power generation and can be deployed in many branches of technology as reviewed by Messerle [1]. MHD generators frequently employ hightemperature working fluids (plasma, gases, superheated sea-water etc) and can substantially enhance conventional thermal power generating system efficiency from $40 \%$ to above $70 \%$ since they recycle a lot of the heat which otherwise in conventional systems would be rejected to the environment. MHD power plants are usually delineated into open and closed cycle based on the nature of processing of the working fluid. With recent expansion into ocean engineering and new developments in naval propulsion and offshore technologies, seawater MHD energy generation has increasingly attracted the attention of engineers and scientists. Examples of new applications of MHD power in recent years include vortex control in helical-channel seawater MHD propulsion for surface vessels [2], coal-fired combustion enhancement [3], hybrid solarMHD astronautical power systems [4], driven disk MHD rocket propulsion [5], fusion Rankine bypass cycles [6], nuclear submarine electromagnetic drives [7] (wherein silent operation at 
much higher speeds is achievable with MHD propulsion systems without encountering cavitation and high turbine blade losses associated with ordinary propellers), commercial ship propulsion [8, 9] and MHD wave energy generation designs [10]. Magnetohydrodynamic (MHD) propulsion and power generation systems function with different physical principles than do conventional propeller drives and industrial energy generators and are therefore not subject to the same physical limits. For example, MHD propulsion relies on the generation of electrical currents in seawater that act in conjunction with relatively large magnetic fields to generate direct thrusts on the seawater via Lorentz magnetic body forces. Effectively the ship and seawater act as the armature and rotor for a linearized electric motor. Similar in nature to electric motors, several modes for MHD propulsion or generators are possible, including induction drive units, AC drives, DC drives etc. Magnetohydrodynamics and heat transfer are central to developing more efficient and sustainable MHD propulsion or generator systems. As identified by Messerle [1], MHD energy generators deploy hot conductive electricallyconducting fluids or gases as the moving conductor. They usually appear in one of three formsthe Faraday generator, the Hall generator, and the disc generator. In recent years all these systems have been studied both experimentally and increasingly with computational simulation. The latter are generally more cost-effective and permit multiple scenarios to be studied with robust mathematical models and numerical methods. Relevant studies in this regard include Arikoglu et al. [11] (who considered the MHD disk generator configuration with slip and entropy effects), Ishikawa et al. [12] (who also considered MHD disk designs in conjunction with carbon dioxide recovery), Lengvel [13] (who considered boundary-layer shorting in Faraday MHD generators) and Stankiewicx and Palmer [14] (who introduced a robust NASA design for the Hall MHD generator). All of these studies have confirmed the superior ability of MHD generators compared with traditional electric generators since the former can perform at much higher temperatures without moving parts. Additionally, in the hot exhaust gas of an MHD generator can heat the boilers of steam plants and therefore attains much higher overall efficiency when deployed as a topping cycle for coal/natural gas burners. Other interesting studies of the fluid dynamics and thermodynamics of MHD power generators include Hardianto et al. [15], Das et al. [16] and Liberati and Okuno [17] which have spanned laminar, turbulent and also stability aspects of operation. As with any other novel technology, a number of issues must be considered in the implementation of an MHD generator and include generator efficiency, economics, sustainability, by-products, scalability, surface degradation, corrosion etc. These issues are affected by the choice of one of the three MHD generator designs. The high temperature intrinsic to MHD generators is known to cause significant 
erosion at the anodes due to chemically-reacting gaseous corrosive environments. Cathode shorts may subsequently result from this and these in turn can also lead to abnormally high current densities on some electrodes and decreased power output [18-20]. These phenomena have motivated researchers to study chemically-reactive magnetohydrodynamic heat transfer in MHD generators in the vicinity of solid surfaces and boundaries (anodes/cathodes) where the effects are substantially greater than in the core duct flow. Viscous hydromagnetic boundary layer theory is a practical tool for building accurate models in these regions of MHD generators. Jena et al. [21] analysed the collective influence of heat sink/source and chemical reaction in boundary-layer magnetic convection with a nonlinear rheological pumping fluid. Thermo-solutal reactive magnetohydrodynamic transport from a vertical boundary with porous media drag, radiative flux and non-Newtonian generator fluid effects was examined by Srinivasacharya and Reddy [22]. Bég et al. [10] used finite difference and network simulation computational methods to analyse optically-dense radiative magnetic boundary layers on an MHD generator wall with suction effects. Although historically the MHD disk generator was believed to be the most efficient MHD power system, recent work has demonstrated the considerable promise of Hall MHD generators, largely following improved wall material designs and novel working fluids which are doped to enhance efficiencies. Faraday generators still remain impracticable since the differential voltages and currents in the fluid short through the electrodes on the sides of the duct and these systems while they do feature a Hall current tend to waste considerable energy resulting in unacceptable efficiencies. This shorting problem is circumvented with the Hall MHD generator wherein the Hall effect is employed to create a current that flows with the fluid. The normal scheme is to the duct. The shorts of the Faraday current induce a powerful magnetic field within the fluid, but in a chord of a circle at right angles to the faraday current and massively decrease losses due to elimination of the shorting of the final induced current. In naval propulsion and ocean generator systems in particular (sweater), Hall current MHD generators hold tremendous potential. This has mobilized a renewed interest in the analysis of different multi-physical phenomena in such generators. Hall currents as noted earlier can have a significant effect on MHD energy systems for example, the presence of longitudinal Hall currents in a flow creates a transverse body force which can lead to transverse pressure gradients, velocity gradients etc. The presence of a Hall current also induces a secondary (cross) flow which requires multi-dimensional fluid dynamic models. The effects of Hall current cannot be neglected especially for ionized fluid media when applied magnetic field strength is strong. In this case the electron cyclotron frequency $(\omega=e \mathrm{M} / \mathrm{m}$, where $e, M$, and $m$ denote the electron charge, the applied magnetic field, and mass of an 
electron, respectively) exceeds the collision frequency so that the electron makes cyclotron orbits between the collisions and will be diverted in a direction mutually perpendicular to the magnetic and electric field directions. Thus, if an electric field is applied perpendicular to the magnetic field then whole current will not pass along the electric field. This complex phenomenon of flow of the electric current across an electric field with an orthogonal magnetic field constitutes the Hall effect, and the associated electrical current is known as the Hall current. Kennedy and Hughes [24] conducted one of the earliest studies of Hall current MHD generator flow wherein they considered computationally the steady state magneto-acoustical response and temporal stability of a ring electrode Hall MHD generator of constant crosssectional area and zero electrode ring slant angle. Murthy [25] investigated Hartmann-Ekman boundary layers. Further studies include Sawaya et al. [26] performed laboratory studies to evaluate the Hall parameter of electrolyte solutions with a closed loop thermosyphonic magnetohydrodynamic circuit under a transverse transverse magnetic field, observing that with excessively high uncontrolled Hall currents, there arises a significant loss in power output at the electrodes due to electron drift in the fluid leading to generation of current in an axial direction at the expense of the current flowing in the transverse direction between the electrodes. Aboeldahab and Elbarbary [27] studied combined heat and mass transfer in Hall current magnetohydrodynamic boundary layers with wall transpiration effects. Bég et al. [28] used an electro-thermal network solver (PSPICE) to determine Ohmic dissipation, ionslip and other effects on time-dependent Hall cross flow in thermo-magnetohydrodynamic HartmannCouette regime. Many other studies have been communicated on Hall current hydromagnetic boundary layers and fully developed duct flows in which significant modifications in both primary and secondary velocity and temperature distributions have been observed with Hall parameter. These include Abo-Eldahab and El Aziz [29] (non-isothermal study), Salem and ElAziz [30] (reactive flow from elastic wall), Bég et al. [31] (rotating Hall plasma MHD generator flow with oblique magnetic field), Aurangzaib et al. [32] (transient thermallystratified reactive flow), Bég et al. [33] (finite element 3-dimensional spinning MHD porous matrix generator flow), Das et al. [34] (buoyancy-driven natural convection), Bég et al. [35] (non-isothermal dissipative Hall current flow), Seth and Singh \{36] (rotating channel with wall electrical conductance) and Kinyanjui et al. [37] (radiative Hall current hydromagnetic moving wall flow).

The corrosive and chemically-reacting nature of near-wall flows in MHD generators, also mobilizes cross-diffusion effects. These include the Soret (thermos-diffusion) effect and the Dufour (diffuso-thermal) effect. The former refers to scenarios in which lower density species 
and higher density species separate at the molecular level under a temperature gradient.In seeded Potassium working fluids which are popular in MHD Hall generators [38], the Soret effect is possible since generally more than one chemical species is present under a very large temperature gradient. In terms of mathematical models, this supplements the species diffusion equation (concentration boundary layer equation) with extra thermal diffusion terms. The Dufour effect describes the energy (heat) flux created when a chemical system is subjected to a significant concentration gradient and may also arise in corrosive boundary layer zones of MHD generators. To simulate this phenomenon, the energy conservation equation has to be augmented with supplementary species diffusion terms. Numerous researchers have investigated Soret and/or Dufour phenomena in both viscous hydrodynamics and magnetohydrodynamics (although generally in the absence of Hall currents in MHD). Representative studies in this regard include Li et al. [39] (who also considered endothermic reactions), Bég et al. [40] (solar collector systems), Gaikwad et al. [41] (non-Newtonian media), Bég et al. [42] (MHD materials processing), Abd El-Aziz (transpiring flows) [43], Bég et al. [44] (micro-morphic thermo-solutal convection from curved bodies), Reddy Sheri et al. [45] (transient hydromagnetic dissipative flow), Pandya and Shukla [46] (high temperature Hall magneto-gas dynamics), Reddy Sheri and Modugula [47] (double-diffusive reactive time-dependent MHD) and Majeed et al. [48] (viscoelastic ferromagnetic boundary layers) The high temperature encountered in MHD Hall generators also invokes thermal radiation heat transfer. This is the most complex mode of heat transfer and requires considerable complexity in numerical methods for accurate simulation. To simplify the integro-differential equation of radiative transfer into a differential equation, algebraic flux models are utilized by engineers. These are particularly advantageous for boundary-layer magnetohydrodynamics. Despite reducing the mathematical complexity (i.e. negation of spectral effects, assumption of constant optical depth, non-scattering and isotropic assumptions), the Rosseland diffusion model remains the most popular of these algebraic radiative flux models. It has been deployed extensively in recent years in many multi-physical radiative engineering flow models. Bég et al. [49] investigated analytically the radiative flux influence on unsteady gravity-driven optically-thick gray convection flow along an inclined plate with pressure gradient. Further studies include Mahmoud [50] (micropolar magnetic flow with thermal conductivity variation), Bég et al. [51] (swirling Von Karman optically-thick MHD transpiring slip flow), El-Hakiem [52] (oscillatory hydromagnetic permeable convection), Shamshuddin et al. [53] (reactive periodic dissipative micropolar thermo-solutal convection), Bég et al. [54] (transient two- 
dimensional convective-radiative hydromagnetics), Hady et al. [55] (radiative nanofluid dynamics) and Bhatti et al. [56] (peristaltic pumping of electromagnetic two-phase flows).

In certain MHD Hall generators, the ducts are inclined to invoke buoyancy and allow variation of the orientation in different surface wave scenarios. The magnetohydrodynamic boundary layer along inclined duct walls is therefore important in particular when corrosive phenomena and cross-diffusion arise during working operations $[57,58]$. The main purpose of the present investigation is therefore to study the composite effects of chemical reaction, viscous dissipation and Hall effects on unsteady MHD thermo-solutal transport from an inclined duct wall (plate) embedded in a porous medium with Soret/Dufour diffusional effects. The porous medium is included as it provides an inert damping mechanism which stabilizes the flow [59]. The governing non-linear partial differential equations for mass, momentum, energy and species conservation are transformed into a system of coupled non-linear ordinary differential equations with appropriate similarity variables. The normalized conservation equations are then solved with a robust finite element method (FEM) subject to corresponding initial and boundary conditions. Solutions are presented for the variation of primary and secondary velocity, temperature, concentration, skin friction, Nusselt number and Sherwood number distributions with Eckert number (dissipation parameter), thermal Grashof number, solutal Grashof number, magnetic body force parameter, Hall parameter, permeability parameter, Dufour number, Soret number, time, radiation-conduction parameter, chemical reaction parameter, heat absorption parameter, Prandt number, Schmidt number and wall inclination. Verification of computations with several special cases from the literature (non-reactive, nondissipative flow in the absence of heat generation or absorption) are included. The simulations are relevant to transport characteristics in the boundary layer region in actual MHD generator systems and provide a useful benchmark for more generalized computational fluid dynamics (CFD) analysis.

\section{HALL MAGNETOHYDRODYNAMIC GENERATOR INCLINED REACTIVE DIFFUSIVE MODEL}

A mathematical model is developed here to simulate near-wall boundary layer transport characteristics in a Hall MHD generator in the context of naval propulsion and/or ocean energy power generation. Figs. 1a,b illustrate the working mechanism of a Hall generator and the principle applied to seawater MHD power generation. A simplification of Fig 1a (as studied in this article) is depicted as the physical configuration in Fig. 1c. We consider the twodimensional, time-dependent magnetohydrodynamic thermo-solutal radiative convection flow 
of a viscous, incompressible electrically-conducting fluid from an inclined infinite porous duct wall (plate).

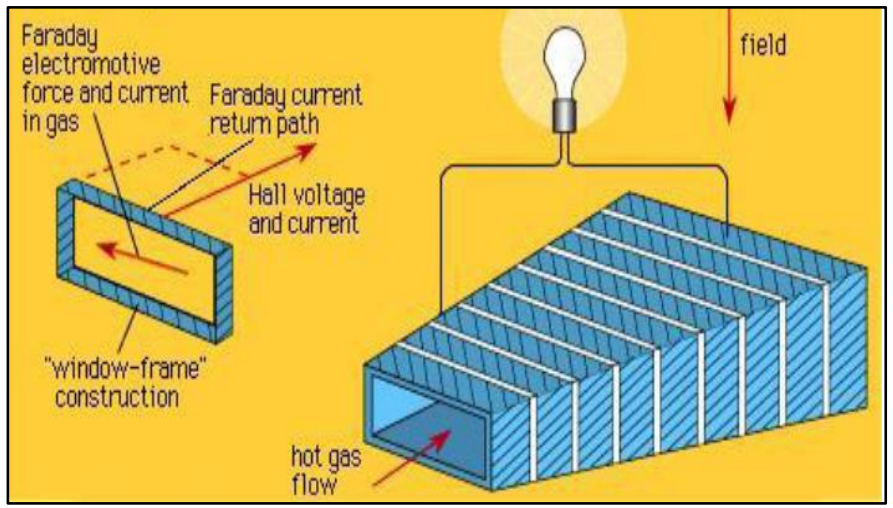

Fig 1a: Hall current MHD generator working mechanism

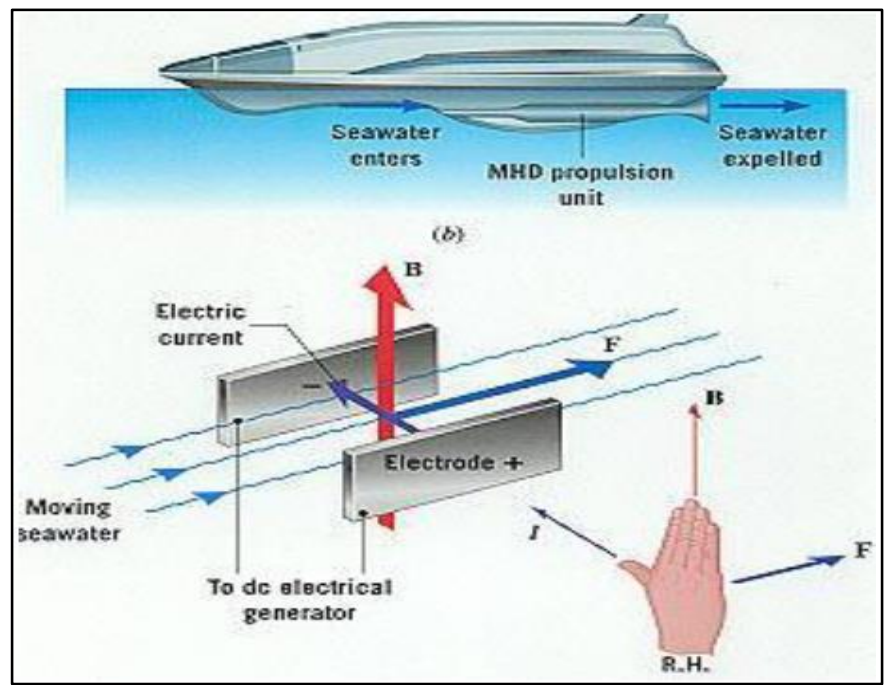

Fig 1b: Ocean MHD Hall current generator propulsion 


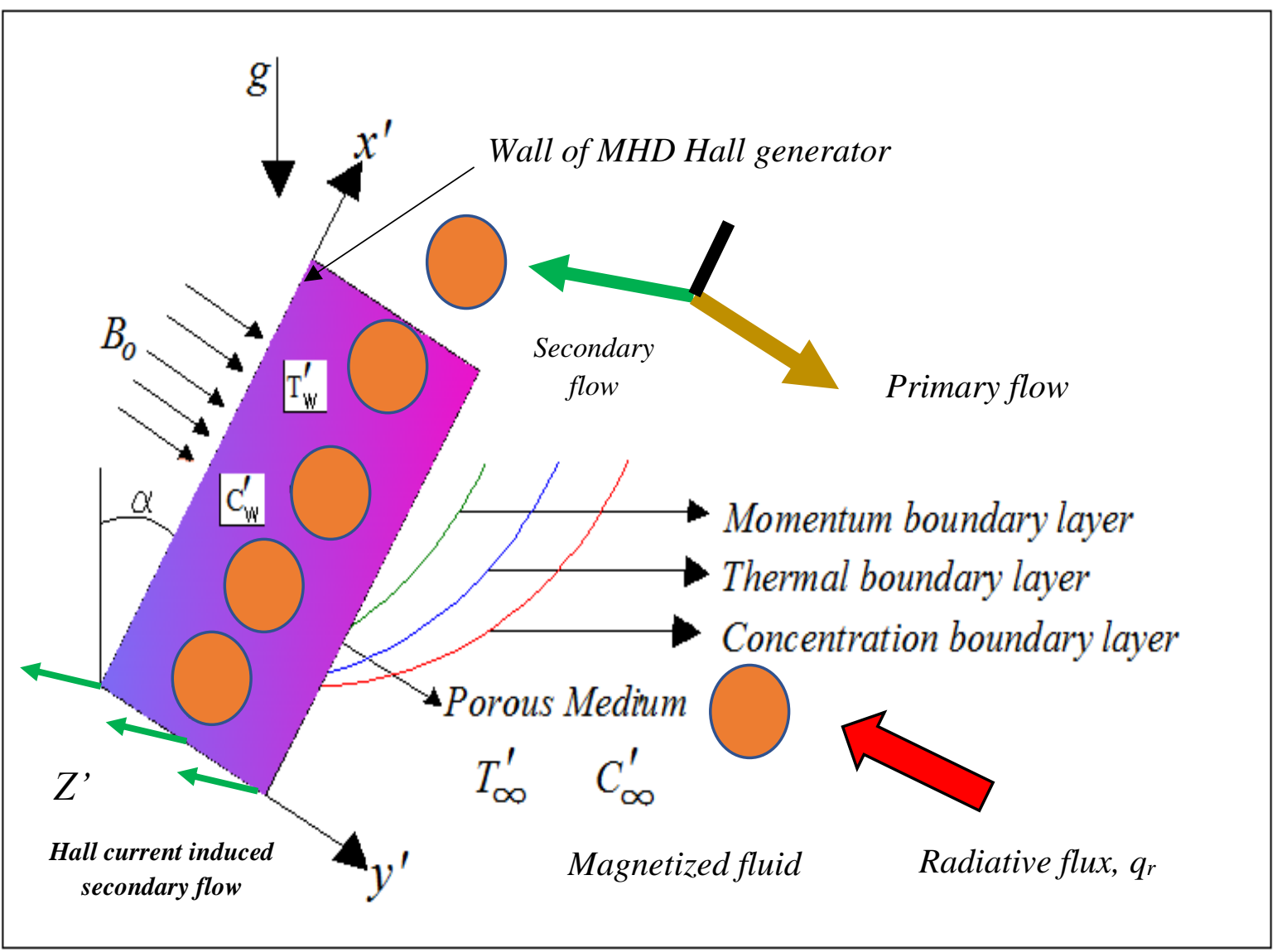

Figure 1c: MHD generator near-wall physical transport model and coordinate system

Soret and Dufour effects are invoked due to density differences in the species (molecular oxygen from corrosion of the duct wall). A transverse magnetic field is applied perpendicular to the plate. The field strength is sufficiently large to generate Hall current which in turn induces a secondary flow in the boundary layer regime. The effect of Hall current gives rise to a force in the $z$-direction, which induces a cross-flow in the $z$-direction and hence the flow becomes three-dimensional. High temperature working conditions invoke significant thermal radiation flux which is assumed uni-directional in nature i.e. radiation heat flux along the $x^{\prime}$ direction is negligible in comparison to the $y^{\prime}$ - direction. The magnetized working fluid is emitting, absorbing but non-scattering in nature and has high optical thickness. Molecular oxygen in the fluid also gives rise to chemical reaction effects and furthermore viscous dissipation and heat sink/source is present. However, Ohmic (Joule) dissipation and magnetic induction effects are negated i.e. the magnetic field is not distorted by fluid vorticity owing to sufficiently low magnetic Reynolds number. Electrical field, Alfven wave, Maxwell displacement and Ohmic heating effects are neglected. The wall temperature and concentration and the free stream temperature and concentration are prescribed as constant i.e. isothermal 
and iso-solutal conditions are enforced. The magnetic fluid contains a reactive species which is modelled by a first order, homogenous, destructive chemical reaction. The MHD generator duct contains a sparsely-packed, isotropic, homogenous, high-permeability porous material. We restrict attention to low Reynolds number, viscous-dominated flow for which Darcy's law is valid. The $x^{\prime}$-axis is orientated along the plate, the $y^{\prime}$ axis is normal to it and the $z^{\prime}$ axis is normal to the $x^{\prime} y^{\prime}$ plane. A uniform magnetic field $B_{0}$ is imposed along the $y^{\prime}$ axis and the MHD generator duct wall (plate) is electrically non-conducting. We assume that all the fluid properties are isotropic and constant. Initially the working fluid and duct wall (plate) are maintained at the same temperature $T_{\infty}^{\prime}$ and concentration $C_{\infty}^{\prime}$. For $t^{\prime}>0$, the duct wall (plate) moves with velocity $u_{0}$, its temperature and concentration are elevated exponentially with time. The $x^{\prime}$ and $y^{\prime}$ components of current density are defined, following Cramer and Pai [60] as follows:

$$
\begin{aligned}
& J_{x}^{\prime}=\frac{\sigma B_{0}}{1+m^{2}}\left(m u^{\prime}-w^{\prime}\right) \\
& J_{z}^{\prime}=\frac{\sigma B_{0}}{1+m^{2}}\left(u^{\prime}+m w^{\prime}\right)
\end{aligned}
$$

Here $u^{\prime}$ and $w^{\prime}$ are the primary and secondary (cross flow) velocities, $J_{x}^{\prime}$ and $J_{z}^{\prime}$ are the electric current densities in the $x^{\prime}$ - and $z^{\prime}$-directions, respectively and $m$ is Hall parameter. Due to the assumed infinite length of the duct wall in the $x^{\prime}$ direction, all flow variables can be regarded as functions of $t^{\prime}$ and $y^{\prime}$ only. Under the usual Boussinesq approximation, governing equations of this problem are given by:

\section{Continuity (mass conservation):}

$$
\frac{\partial v^{\prime}}{\partial y^{\prime}}=0 \Rightarrow v^{\prime}=-v_{0}(\text { cons } \tan t)
$$

\section{Momentum conservation:}

\section{Primary}

$$
\frac{\partial u^{\prime}}{\partial t^{\prime}}+v^{\prime} \frac{\partial u^{\prime}}{\partial y^{\prime}}=v \frac{\partial^{2} u^{\prime}}{\partial y^{\prime 2}}+g \gamma\left(T^{\prime}-T_{\infty}^{\prime}\right) \cos (\alpha)+g \gamma^{*}\left(C^{\prime}-C_{\infty}^{\prime}\right) \cos (\alpha)-\frac{\sigma B_{0}^{2}}{\rho\left(1+m^{2}\right)}\left(u^{\prime}+m w^{\prime}\right)-\frac{v u^{\prime}}{K^{\prime}}
$$




\section{Secondary (Cross flow)}

$$
\frac{\partial w^{\prime}}{\partial t^{\prime}}+v^{\prime} \frac{\partial w^{\prime}}{\partial y^{\prime}}=v \frac{\partial^{2} w^{\prime}}{\partial y^{\prime 2}}+\frac{\sigma B_{0}^{2}}{\rho\left(1+m^{2}\right)}\left(m u^{\prime}-w^{\prime}\right)-\frac{v w^{\prime}}{K^{\prime}}
$$

\section{Energy (heat) conservation:}

$\left(\frac{\partial T^{\prime}}{\partial t^{\prime}}+v^{\prime} \frac{\partial T^{\prime}}{\partial y^{\prime}}\right)=\frac{1}{\rho c_{p}}\left(k \frac{\partial^{2} T^{\prime}}{\partial y^{\prime 2}}-\frac{\partial q_{r}}{\partial y^{\prime}}+\frac{\rho D_{m} K_{T}}{c_{s}} \frac{\partial^{2} C^{\prime}}{\partial y^{\prime 2}}\right)+\mu\left(\frac{\partial u^{\prime}}{\partial y^{\prime}}\right)^{2}-Q_{0}\left(T^{\prime}-T_{\infty}^{\prime}\right)$

Species (molecular reacting material) conservation:

$$
\frac{\partial C^{\prime}}{\partial t^{\prime}}+v^{\prime} \frac{\partial C^{\prime}}{\partial y^{\prime}}=D \frac{\partial^{2} C^{\prime}}{\partial y^{\prime 2}}+\frac{D_{m} K_{T}}{T_{m}} \frac{\partial^{2} T^{\prime}}{\partial y^{\prime 2}}-k_{r}\left(C^{\prime}-C_{\infty}^{\prime}\right)
$$

Here $\gamma^{*}$ is the coefficient of volume expansion for mass transfer, $\gamma$ is volumetric coefficient of thermal expansion, $v^{\prime}$ is velocity along $y^{\prime}$ axis, $w^{\prime}$ is velocity along $z^{\prime}$-axis, $K^{\prime}$ is permeability of the porous medium, $\sigma$ is electrical conductivity of the magnetic fluid, $B_{o}$ is transverse magnetic field, $D_{m}$ is molecular diffusivity of the reactive species (e.g. oxygen), $g$ is acceleration due to gravity, $K_{T}$ is thermal diffusion ratio, $\mu$ is the viscosity, $\rho$ is the magnetic fluid density, $k$ is thermal conductivity of fluid, $C^{\prime}$ and $T^{\prime}$ are dimensional concentration and temperature, $C_{\infty}^{\prime}$ and $T_{\infty}^{\prime}$ are concentration and temperature of free stream, $k_{r}$ is chemical reaction parameter, the term $Q_{0}\left(T^{\prime}-T_{\infty}^{\prime}\right)$ is considered to be amount of heat generated or absorbed per unit volume, $Q_{0}$ is the constant, for heat source $Q_{0}>0$ and for heat sink $Q_{0}<0$ ; $c_{p}$ is specific heat at constant pressure, $q_{r}$ is radiative heat along $y^{\prime}$-axis, $v$ is kinematic viscosity of the magnetic fluid and $T_{m}$ is mean fluid temperature.

The following boundary and initial conditions are prescribed:

$$
\begin{aligned}
& t^{\prime} \leq 0: \quad u^{\prime}=0, w^{\prime}=0, T^{\prime}=T_{\infty}^{\prime}, C^{\prime}=C_{\infty}^{\prime} \quad \text { forall } y^{\prime} \\
& t^{\prime}>0: \quad u^{\prime}=u_{0}, v^{\prime}=-v_{0}, \quad w^{\prime}=0, T^{\prime}=T_{\infty}^{\prime}+\left(T_{w}^{\prime}-T_{\infty}^{\prime}\right) e^{B t^{\prime}}, \\
& C^{\prime}=C_{\infty}^{\prime}+\left(C_{w}^{\prime}-C_{\infty}^{\prime}\right) e^{B t^{\prime}} \quad \text { at } \quad y^{\prime}=0 \\
& u^{\prime}=0, \quad w^{\prime}=0, \quad T^{\prime} \rightarrow T_{\infty}^{\prime}, \quad C^{\prime} \rightarrow C_{\infty}^{\prime} \quad \text { as } \quad y^{\prime} \rightarrow \infty .
\end{aligned}
$$


Here $T_{w}^{\prime}$ and $C_{w}^{\prime}$ denote temperature and concentration at the duct wall (plate) surface and $B=\frac{v_{0}^{2}}{v}$

The radiative heat flux term is defined using the Rosseland diffusion approximation:

$q_{r}=-\left(4 \sigma^{*} / 3 k_{m}\right)(\partial T \quad / 4 / \partial y \quad /)$

Here $\sigma *$ and $k_{m}$ are Stefan Boltzmann constant and mean absorption coefficient, respectively. This formulation allows the transformation of the governing integro-differential equation for radiative energy balance into electrostatic potential (Coulomb's law) which is valid for optically-thick media in which radiation only propagates a limited distance prior to experiencing scattering or absorption. It can be shown that the local intensity is caused by radiation emanating from nearby locations in the vicinity of which the emission and scattering are comparable to the location under consideration. For zones where conditions are appreciably different, the radiation has been shown to be greatly attenuated prior to arriving at the location being analyzed. The energy transfer depends only on the conditions in the area near the position under consideration. In applying the Rosseland assumption, it is assumed that refractive index of the medium is constant, intensity within the porous medium is nearly isotropic and uniform and wavelength regions exist where the optical thickness is greater than 5. The temperature difference within the flow is very small, so that $T^{\prime 4}$ may be expressed linearly with temperature. It is observed by expanding in a Taylor's series about $T_{\infty}^{\prime}$ and considering negligible higher order terms, the following relation emerges:

$T^{\prime 4} \cong 4 T_{\infty}^{\prime 3} T^{\prime}-3 T_{\infty}^{\prime 4}$

By virtue of Eqns. (8) and (9), Eqn. (5) is reduced to:

$$
\rho c_{p}\left(\frac{\partial T^{\prime}}{\partial t^{\prime}}+v^{\prime} \frac{\partial T^{\prime}}{\partial y^{\prime}}\right)=k \frac{\partial^{2} T^{\prime}}{\partial y^{\prime 2}}+\frac{16 \sigma T_{\infty}^{\prime 3}}{3 k_{m}} \frac{\partial^{2} T^{\prime}}{\partial y^{\prime 2}}+\frac{\rho D_{m} K_{T}}{c_{s}} \frac{\partial^{2} C^{\prime}}{\partial y^{\prime 2}}+\mu\left(\frac{\partial u^{\prime}}{\partial y^{\prime}}\right)^{2}-Q_{0}\left(T^{\prime}-T_{\infty}^{\prime}\right)
$$

In order to obtain the non-dimensional form of the governing equations, we introduce the following quantities: 


$$
\begin{aligned}
& u=\frac{u^{\prime}}{u_{0}}, t=\frac{t^{\prime} v_{0}^{2}}{v}, \theta=\frac{T^{\prime}-T_{\infty}^{\prime}}{T_{w}^{\prime}-T_{\infty}^{\prime}}, C=\frac{C^{\prime}-C_{\infty}^{\prime}}{C_{w}^{\prime}-C_{\infty}^{\prime}}, G m=\frac{v g \gamma^{*}\left(C_{w}^{\prime}-C_{\infty}^{\prime}\right)}{u_{0} v_{0}^{2}}, \\
& G r=\frac{v g \gamma\left(T_{w}^{\prime}-T_{\infty}^{\prime}\right)}{u_{0} v_{0}^{2}}, \quad D u=\frac{D_{m} K_{T}\left(C_{w}^{\prime}-C_{\infty}^{\prime}\right)}{c_{s} c_{p} v\left(T_{w}^{\prime}-T_{\infty}^{\prime}\right)}, \quad S r=\frac{D_{m} K_{T}\left(T_{w}^{\prime}-T_{\infty}^{\prime}\right)}{T_{m} v\left(C_{w}^{\prime}-C_{\infty}^{\prime}\right)}, \\
& K=\frac{v_{0}^{2} K^{\prime}}{v^{2}}, \quad \operatorname{Pr}=\frac{\mu c_{p}}{k}, M=\frac{\sigma B_{0}^{2} v}{\rho v_{0}^{2}}, \quad S c=\frac{v}{D_{m}}, \quad R=\frac{4 \sigma T_{\infty}^{\prime 3}}{k_{m} k}, \\
& y=\frac{y^{\prime} v_{0}}{v}, \quad w=\frac{w^{\prime}}{u_{0}}, \quad E c=\frac{u_{0}^{2}}{c_{p}\left(T_{w}^{\prime}-T_{\infty}^{\prime}\right)}, \quad K_{r}=\frac{k_{r} v}{v_{0}^{2}}, \quad Q=\frac{Q_{0} v}{\rho c_{p} v_{0}^{2}} .
\end{aligned}
$$

All parameters are defined in the nomenclature. Introducing (11) into Eqns. (2), (3), (4), (10), (6), the following normalized partial differential equations are produced:

$$
\frac{\partial u}{\partial t}-\frac{\partial u}{\partial y}=\frac{\partial^{2} u}{\partial y^{2}}+G r \cos (\alpha) \theta+G m \cos (\alpha) C-\left(\frac{M}{1+m^{2}}+\frac{1}{K}\right) u-\left(\frac{m M}{1+m^{2}}\right) w
$$

$$
\begin{aligned}
& \frac{\partial w}{\partial t}-\frac{\partial w}{\partial y}=\frac{\partial^{2} w}{\partial y^{2}}-\left(\frac{M}{1+m^{2}}+\frac{1}{K}\right) w+\left(\frac{m M}{1+m^{2}}\right) u \\
& \frac{\partial \theta}{\partial t}-\frac{\partial \theta}{\partial y}=\frac{1}{\operatorname{Pr}}\left(1+\frac{4 R}{3}\right) \frac{\partial^{2} \theta}{\partial y^{2}}+D u \frac{\partial^{2} C}{\partial y^{2}}+E c\left(\frac{\partial u}{\partial y}\right)^{2}-Q \theta \\
& \frac{\partial C}{\partial t}-\frac{\partial C}{\partial y}=\frac{1}{S c} \frac{\partial^{2} C}{\partial y^{2}}+S r \frac{\partial^{2} \theta}{\partial y^{2}}-K_{r} C
\end{aligned}
$$

The non-dimensional boundary and initial conditions (7) now assume the form:

$$
\begin{aligned}
& t \leq 0 \quad u=0 \quad w=0 \quad \theta=0 \quad C=0 \quad \forall y \\
& t>0 \quad u=1 \quad w=0 \quad \theta=e^{t} \quad C=e^{t} \quad \text { at } \quad y=0 \\
& u=0 \quad w=0 \quad \theta \rightarrow 0 \quad C \rightarrow 0 \quad y \rightarrow \infty
\end{aligned}
$$

Important engineering design quantities in MHD power and propulsion systems are the skinfriction coefficients $\tau_{1}$ and $\tau_{2}$ along the $x^{\prime}$-axis and $z^{\prime}$-axis, respectively, the Nusselt number $N u$ (heat transfer rate at the wall) and the Sherwood number $S h$ (species gradient at the wall). The non-dimensional forms of these physical quantities are:

$$
\tau_{1}=\left(\frac{\partial u}{\partial y}\right)_{y=0}, \tau_{2}=\left(\frac{\partial w}{\partial y}\right)_{y=0}, N u=-\left(\frac{\partial \theta}{\partial y}\right)_{y=0}, S h=-\left(\frac{\partial C}{\partial y}\right)_{y=0}
$$




\section{COMPUTATIONAL SOLUTION WITH FINITE ELEMENT METHOD}

The transformed system of dimensionless, non-linear, coupled, non-homogeneous partial differential equations (12) - (15) under the initial/boundary conditions (16) is solved numerically using the extensively validated and robust finite element method (FEM) with a Galerkin weighted residual scheme. This method comprises five fundamental steps, namely discretization of the domain, derivation of the element equations, assembly of element equations, imposition of boundary conditions and finally deployment of an iterative scheme which is employed to solve the matrix system (e.g. Gaussian elimination method) maintaining an accuracy of $10^{-7}$. The FEM code is run in the MATLAB symbolic software. Comprehensive details of this methodology are available for magnetofluid dynamics in Bég [61]. A more general discourse is provided in the excellent monograph of Reddy [62]. The fundamental flow/heat/mass transfer characteristics are computed i.e. primary and secondary velocity profiles, temperature, reactive species concentration profile, primary and secondary skinfriction coefficients, Nusselt number and Sherwood number with variation in the numerous thermal/magnetic/chemical/hydrodynamic parameters defined in Eqn. (11).

\section{GRID INDEPENDENCE STUDY}

In any numerical method utilizing grids (meshes) it is necessary to conduct a grid-independence test to ensure that the mesh is adequately refined to provide the best, fully converged solutions. The grid independence is conducted by dividing the entire domain into successively sized grids $61 \times 61,81 \times 81,101 \times 101$ and $121 \times 121$. For all the computations, 101 intervals of equal length 0.01 are considered. At each node three functions are to be evaluated. Therefore, after assembly of these elements a system of 303 non-linear equations are formed. Thereafter an iterative scheme is adopted and by introducing boundary conditions, the resulting system of linear algebraic equations is solved. In order to prove the convergence and stability of finite element method the MATLAB-based code is compiled with slightly modified values of velocity profiles $(u, w)$, temperature profile $(\theta)$ and reactive species concentration profile $(C)$ - no significant change was observed in the values of velocity profiles $u, w$, temperature profile $\theta$ and concentration profile $C$. This process was repeated until the desired accuracy of $10^{-7}$ had been obtained. Hence, the finite element MATLAB code is confirmed to be stable and convergent. 


\section{VALIDATION OF NUMERICAL RESULTS}

In order to validate the accuracy of the numerical results obtained through the MATLAB FEM code, the present results are compared with the results reported by Pandya and Shukla [46] in the absence of chemical reaction, viscous dissipation and heat absorption (i.e. $K r=0, E c=0$, and $Q=0)$. The effects of $D u, K, M, m, S c, S r, \alpha$ on the primary and secondary skin-friction coefficients, Nusselt number and Sherwood number are documented in Table 1 and Table 2 and evidently exhibit excellent agreement with the results reported by Pandya and Shukla [46] Therefore, the developed MATLAB-FEM numerical code can be used with a great confidence. From Table 1, it is observed that, primary and secondary skin-friction coefficients $\tau_{1}$ and $\tau_{2}$ along the $x^{\prime}$-axis and $z^{\prime}$-axis increase with an elevation in Dufour number, Hall parameter and Soret number (i.e. $D u, m, S r$ ) whereas they are suppressed with a rise in magnetic body force parameter and wall inclination $(M, \alpha)$. Furthermore, there is a significant reduction in primary and secondary skin-friction coefficients $\left(\tau_{1}\right.$ and $\left.\tau_{2}\right)$ with a decrease in permeability parameter $(K)$ and an increase in Schmidt number $(S c)$. From Table 2, it is apparent that as Dufour number $(\mathrm{Du})$ and Soret number $(\mathrm{Sr})$ increase, the Nusselt number $N u$ increases whereas the Sherwood number Sh decreases. However, as Schmidt number is raised, the Nusselt number $N u$ decreases whereas the Sherwood number $S h$ increases.

Table 1: Comparison of Skin friction coefficients $\left(\tau_{1}\right.$ and $\left.\tau_{2}\right)$ with $K r=0, E c=0$ and $Q=0$

\begin{tabular}{|c|c|c|c|c|c|c|c|c|c|c|}
\hline \multirow{2}{*}{$D u$} & \multirow{2}{*}{$K$} & \multirow{2}{*}{$M$} & \multirow{2}{*}{$m$} & \multirow{2}{*}{$S c$} & \multirow{2}{*}{$S r$} & \multirow{2}{*}{$\alpha$} & \multicolumn{2}{|c|}{$\begin{array}{c}\text { Pandya and Shukla } \\
{[46]}\end{array}$} & \multicolumn{2}{|c|}{$\begin{array}{l}\text { MATLAB FEM } \\
\text { code }\end{array}$} \\
\hline & & & & & & & $\tau_{1}$ & $\tau_{2}$ & $\tau_{1}$ & $\tau_{2}$ \\
\hline 0.2 & 2 & 6 & 0.2 & 1 & 2 & 35 & 0.919244 & 0.283318 & 0.919242 & 0.283315 \\
\hline 0.3 & 2 & 6 & 0.2 & 1 & 2 & 35 & 0.943915 & 0.283680 & 0.943916 & 0.283679 \\
\hline 0.2 & 0.5 & 6 & 0.2 & 1 & 2 & 35 & 0.266596 & 0.254016 & 0.266595 & 0.254015 \\
\hline 0.2 & 2 & 1 & 0.2 & 1 & 2 & 35 & 2.03011 & 0.067415 & 2.03012 & 0.067415 \\
\hline 0.2 & 2 & 6 & 0.8 & 1 & 2 & 35 & 1.08976 & 0.821369 & 1.08977 & 0.821369 \\
\hline
\end{tabular}




\begin{tabular}{|l|c|c|c|c|c|c|c|c|c|c|}
\hline 0.2 & 2 & 6 & 0.2 & 3 & 2 & 35 & 0.123565 & 0.25864 & 0.123565 & 0.25863 \\
\hline 0.2 & 2 & 6 & 0.2 & 1 & 4 & 35 & 0.922489 & 0.302472 & 0.922488 & 0.302471 \\
\hline 0.2 & 2 & 6 & 0.2 & 1 & 2 & 15 & 1.19466 & 0.306231 & 1.19465 & 0.306232 \\
\hline
\end{tabular}

Table 2. Comparison of Nusselt number $(N u)$ and Sherwood number $(S h)$ when $K r=0, E c=0$ and $Q=0$.

\begin{tabular}{|c|c|c|c|c|c|c|c|c|}
\hline \multirow{2}{*}{$D u$} & \multirow{2}{*}{ Sc } & \multirow{2}{*}{$S r$} & \multicolumn{2}{|c|}{$\begin{array}{c}\text { Pandya and Shukla } \\
\qquad[46]\end{array}$} & \multicolumn{2}{|c|}{ MATLAB FEM code } & \multicolumn{2}{|c|}{ MS-DTM algorithm } \\
\hline & & & $N u$ & $S h$ & $N u$ & $S h$ & $N u$ & $S h$ \\
\hline 0.2 & 1 & 2 & 2.48536 & -0.671419 & 2.48535 & -0.671418 & 2.48544 & -0.671408 \\
\hline 0.3 & 1 & 2 & 2.6906 & -1.04464 & 2.6905 & -1.04462 & 2.69101 & -1.04459 \\
\hline 0.4 & 1 & 2 & 3.0872 & -1.76321 & 3.0871 & -1.76320 & 3.0874 & -1.76324 \\
\hline 0.2 & 3 & 2 & 0.68002 & 3.44431 & 0.68001 & 3.44432 & 0.68012 & 3.44454 \\
\hline 0.2 & 6 & 2 & 0.634183 & 5.12367 & 0.634184 & 5.12368 & 0.634221 & 5.12373 \\
\hline 0.2 & 8 & 2 & 0.610059 & 6.00334 & 0.610058 & 6.00335 & 0.610061 & 6.00341 \\
\hline 0.2 & 1 & 4 & 0.735019 & 1.35892 & 0.735018 & 1.35893 & 0.735023 & 1.35897 \\
\hline 0.2 & 1 & 6 & 0.747922 & 0.843116 & 0.747921 & 0.843115 & 0.747926 & 0.843122 \\
\hline 0.2 & 1 & 9 & 0.769676 & -0.0196965 & 0.769677 & -0.0196964 & 0.769677 & -0.019697 \\
\hline
\end{tabular}

\section{FURTHER VALIDATION WITH MULTI-STEP DTM ALGORITHM "MS-DTM"}

Since the present model is novel there are no existing solutions in the literature with which validation of the general model can be conducted. We therefore use an alternative approach and validate the solutions with an alternative numerical method known as the multi-step differential transform method (MS-DTM) developed by Zhou [63] originally for electrical 
circuit analysis. This technique has been used successfully to simulate numerous multi-physical magnetohydrodynamic flow problems including entropy generation minimization in swirling magnetic propulsion systems [64]. DTM is an extremely versatile technique which avoids the need for symbolic computation. DTM has a strong advantage in that it can be applied directly to differential equations without requiring linearization, discretization or perturbation. Another important advantage is that this method reduces the size of computational work while the Taylor series method is computationally exhaustive for large orders. DTM is different from the traditional higher order Taylor series method, the latter requiring symbolic computation and thereby causing greater computationally expense for large orders. However, the DTM obtains a polynomial series solution by means of an iterative procedure. DTM is therefore a robust alternative procedure for obtaining analytic Taylor series solution of linear or nonlinear multidegree, multi-order ordinary or partial differential equations. Table 3 summarizes the fundamental transforms and their associated functions. We provide a brief description of DTM next. The transformation of the $k$ th derivative of a function in one variable is as follows [50]:

$$
F(k)=\frac{1}{k !}\left[\frac{d^{k} f(t)}{d t^{k}}\right]_{t=t_{0}},
$$

and the inverse transformation is defined by

$$
f(t)=\sum_{k=0}^{\infty} F(k)\left(t-t_{0}\right)^{k}
$$

Eqns. (18) and (19) lead to:

$$
f(t)=\left.\sum_{k=0}^{\infty} \frac{\left(t-t_{0}\right)^{k}}{k !} \frac{d^{k} f(t)}{d t^{k}}\right|_{t=t_{0}},
$$

This demonstrates that the concept of differential transform method results from a Taylor series expansion. However, the method does not compute the derivatives representatively. Instead relative derivatives are calculated by an iterative approach which is described by the transformed equations of the original function. For computational purposes, the function $f(t)$ is expressed by a finite series and Eq. (19) can be written as follows: 


$$
f(t) \cong \sum_{k=0}^{i} F(k)\left(t-t_{0}\right)^{k}
$$

where $F(k)$ is the differential transform of $f(t)$.

In the multi-step Differential Transform Method (MDTM), the above concept is extended to equally spaced nodal points $t_{i} ; 0=t_{0}<t_{1}<\cdots<t_{N-1}<t_{N}=b$ and $\left(t_{i+1}-t_{i}\right)=b / N$. On the $\mathrm{i}^{\text {th }}$ subdomain we assume:

$$
\left(t_{i}, t_{i+1}\right) \equiv D_{i},\left.f(t)\right|_{D_{i}}=f_{i}(t)
$$

The differential transform $F_{i}(k)$ of $f_{i}(t)$ at $t=t_{i}$ is then defined by:

$$
F_{i}(k)=\frac{1}{k !}\left[\frac{d^{k} f_{i}(t)}{d t^{k}(t)}\right]_{t=t_{i}} .
$$

The differential inverse transform of $F_{i}(k)$ is defined by

$$
f_{i}(t)=\sum_{k=0}^{\infty} F_{i}(k)\left(t-t_{i}\right)^{k}
$$

Let $f_{i, n}(t)$ be defined by the finite term Taylor series

$$
f_{i, n}(t)=\sum_{k=0}^{n} F_{i}(k)\left(t-t_{i}\right)^{k} \equiv s_{i, n}(t)
$$

It is an important consideration to provide the initial condition $f_{i, n}(t)$ at each $t=t_{i}, i=0,1, \cdots, N$. Since $f(0)=a, f^{\prime}(0)=0$ are given by the initial condition, we have $F_{0}(0)=a, F_{0}(1)=0$. On each $i^{t h}(i \geq 1)$ sub-interval, we approximate $f_{i, n}\left(t_{i}\right)$ and $f_{i, n}\left(t_{i}\right)$ by computing $s_{i-1, n}\left(t_{i}\right)$ and $s_{i-1, n}^{\prime}\left(t_{i}\right)$, respectively. In other words:

$$
\begin{aligned}
& f_{i, n}\left(t_{i}\right)=\sum_{k=0}^{n} F_{i-1}(k)\left(t_{i}-t_{i-1}\right)^{k}, \\
& f^{\prime}{ }_{i, n}\left(t_{i}\right)=\sum_{k=1}^{n} F_{i-1}(k) k\left(t_{i}-t_{i-1}\right)^{k-1} .
\end{aligned}
$$

Table-3: DTM Transformations 


\begin{tabular}{|l|l|}
\hline Original function & Transformed function \\
\hline$f(x)=g(x) \pm h(x)$ & $F(k)=G(k) \pm H(k)$ \\
\hline$f(x)=\operatorname{cg}(x)$ & $F(k)=c G(k)$ \\
\hline$f(x)=\frac{d^{n} g(x)}{d x^{n}}$ & $F(k)=\frac{(k+n) !}{k !} G(k+n)$ \\
\hline$f(x)=g(x) h(x)$ & $F(k)=\sum_{l=0}^{k} G(l) H(k-l)$ \\
\hline$f(x)=x^{n}$ & $F(k)=\delta(k-n)=\left\{\begin{array}{l}1, k=n \\
0, k \neq n\end{array}\right.$ \\
\hline
\end{tabular}

The last two columns in Table 2 provide a comparison with the MS-DTM approach and both the MATLAB FEM solutions and those of Pandya and Shukla [46] for the non-reactive, nondissipative case without heat absorption (i.e. $K r=0, E c=0, Q=0$ ). Excellent agreement is attained. Computations take seconds to compile on a four quad Desk Top machine. To validate with the general model developed in this article, we have benchmarked with the MATLAB FEM solutions in Figs 2-3 for the primary and secondary velocities and species concentration where again very close correlation is attained. The present MATLAB-FEM code is therefore very accurate and provides a further benchmark for extension to more complex flow models by other researchers.

\section{RESULTS AND DISCUSSION}

In order to elucidate the characteristics of the hydrodynamic, heat and mass transfer processes in the near-wall (boundary-layer regime) of the MHD wall generator duct, it is necessary to conduct a parametric study. In this context, we present extensive numerical results in Figs 226 for primary and secondary velocity profiles $(u, w)$, temperature profiles $(\theta)$ and the reactive species concentration profiles $(C)$ for various values of the emerging dimensionless magnetic/thermal/hydrodynamic/reactive parameters. The following parameter values are adopted for computations unless otherwise indicated in the figures and tables. $G r=6.0, G m=$ 
11.0, $M=6.0, K=2.0, \operatorname{Pr}=0.7, R=3.0, E c=2.0, S r=2.0, D u=2.0, S c=0.2, K r=2.0, t=$ $0.2, m=0.2, Q=3.0$.

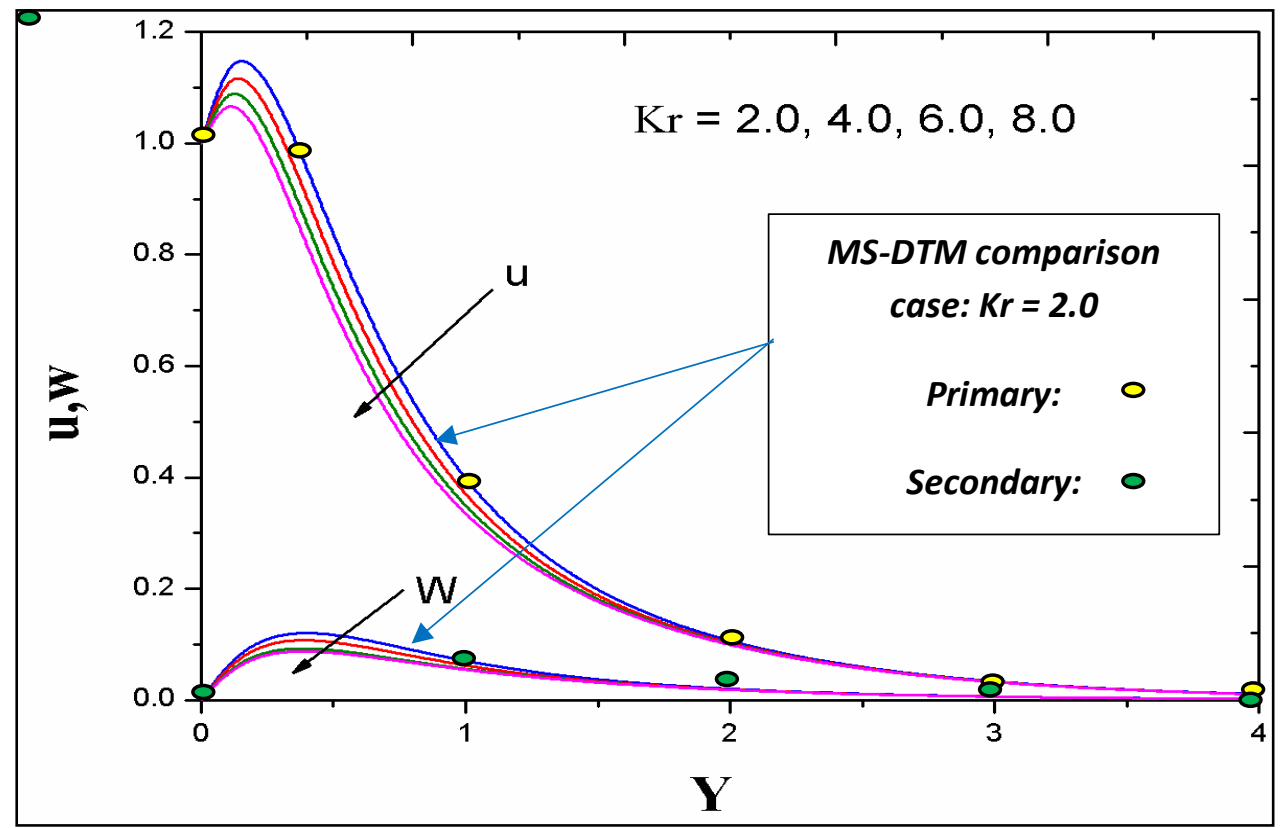

Figure 2: Primary and secondary velocity distributions with reaction parameter $(K r)$

Figure 2 visualizes the influence of the chemical reaction parameter $(K r)$ on primary and secondary velocity fields. Here, as the value of $K r$ increases both primary and secondary (cross flow) velocity magnitudes are markedly decreased. There are two major classifications of chemical reactions, namely homogeneous and heterogeneous. Chemical changes occurring with liquids or gases depend on the type of interactions of these chemical substances. Homogeneous reactions occur in one phase only whereas heterogeneous reactions occur in two or more phases. These types of reaction arise frequently during operations in MHD generators [65]. The destruction of molecular species in the boundary layer via reaction inhibits the momentum diffusion which results in significant flow deceleration and an increase in primary momentum boundary layer and secondary momentum boundary layer thicknesses. Significantly larger magnitudes are computed for the primary velocity $(u)$ as compared with the secondary velocity $(w)$. Additionally, there is a strong velocity overshoot near the duct wall surface arising in the primary flow which is substantially stifled in the secondary flow. These distributions concur with the trends reported in Cramer and Pai [60] among others. Stronger chemical reaction is therefore clearly counter-productive and will inevitably lead to decreasing power efficiencies, as noted by Pollina et al. [65] who have further highlighted that the combination of high temperature, gap voltage, magnetic field intensity and chemical corrosion of wall duct materials reduces the lifetimes of MHD Hall generators. 


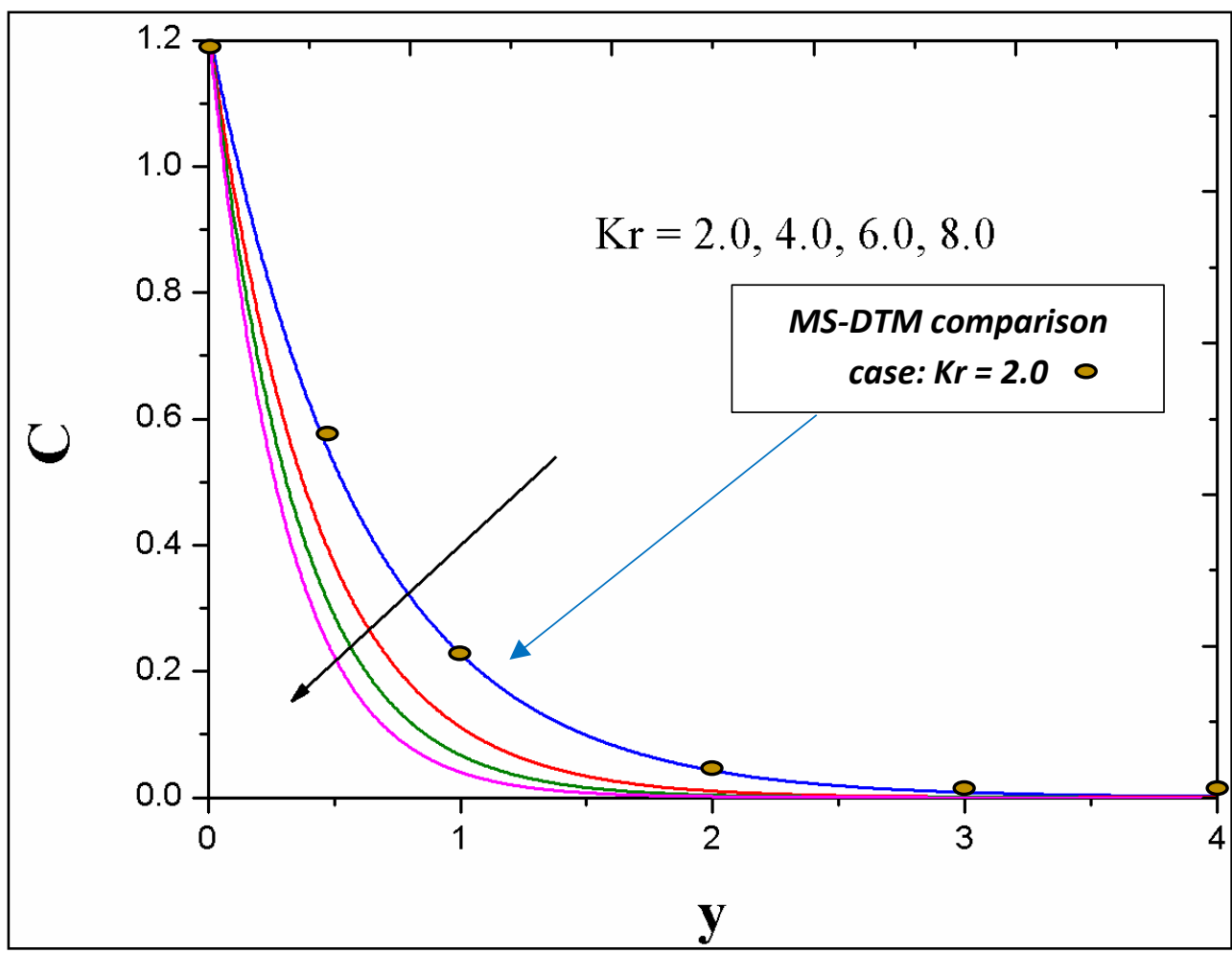

Figure 3: Concentration profiles for different values of reaction parameter $(\mathrm{Kr})$

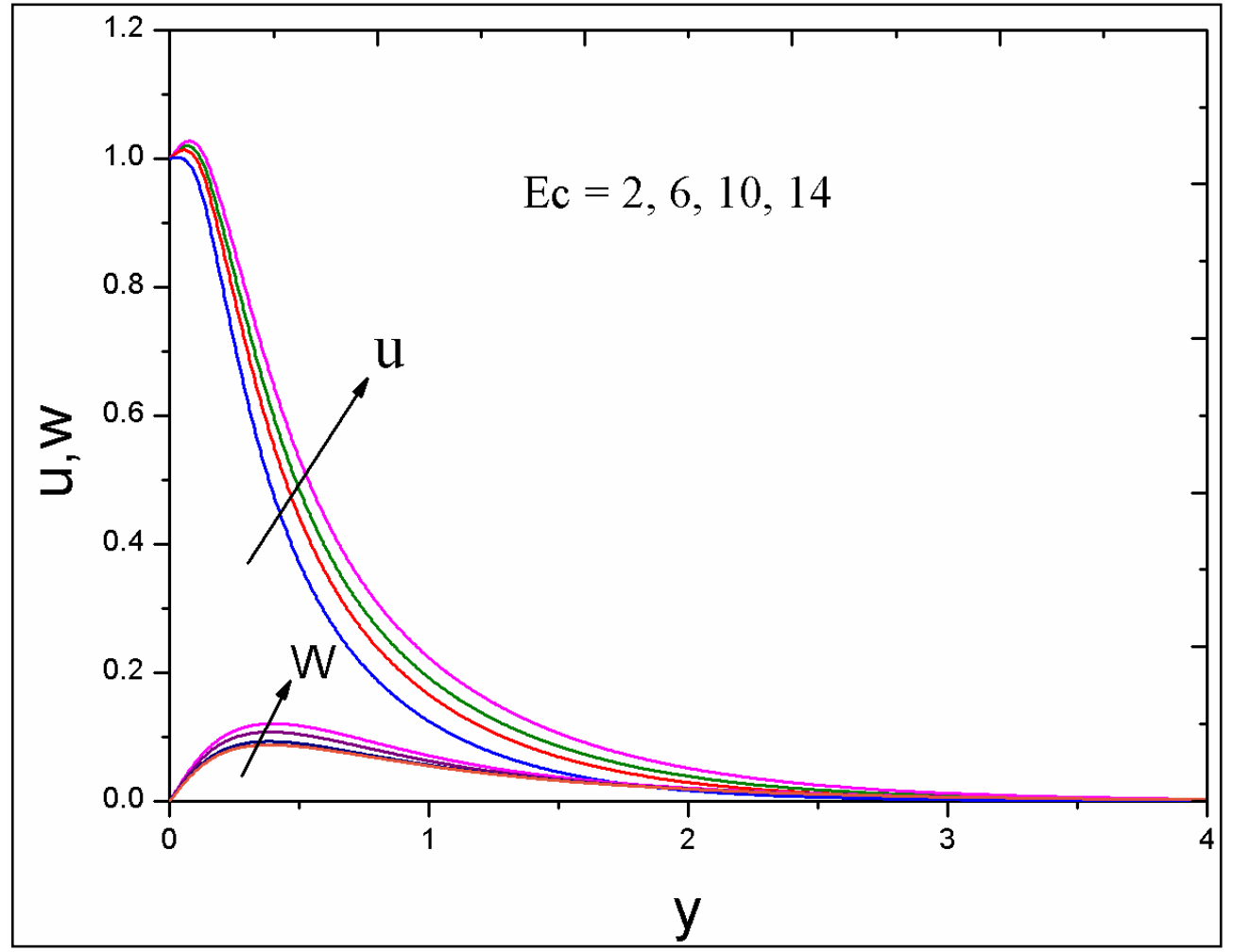

Figure 4: Primary and secondary velocity distributions with Eckert (viscous heating) number $(E c)$. 


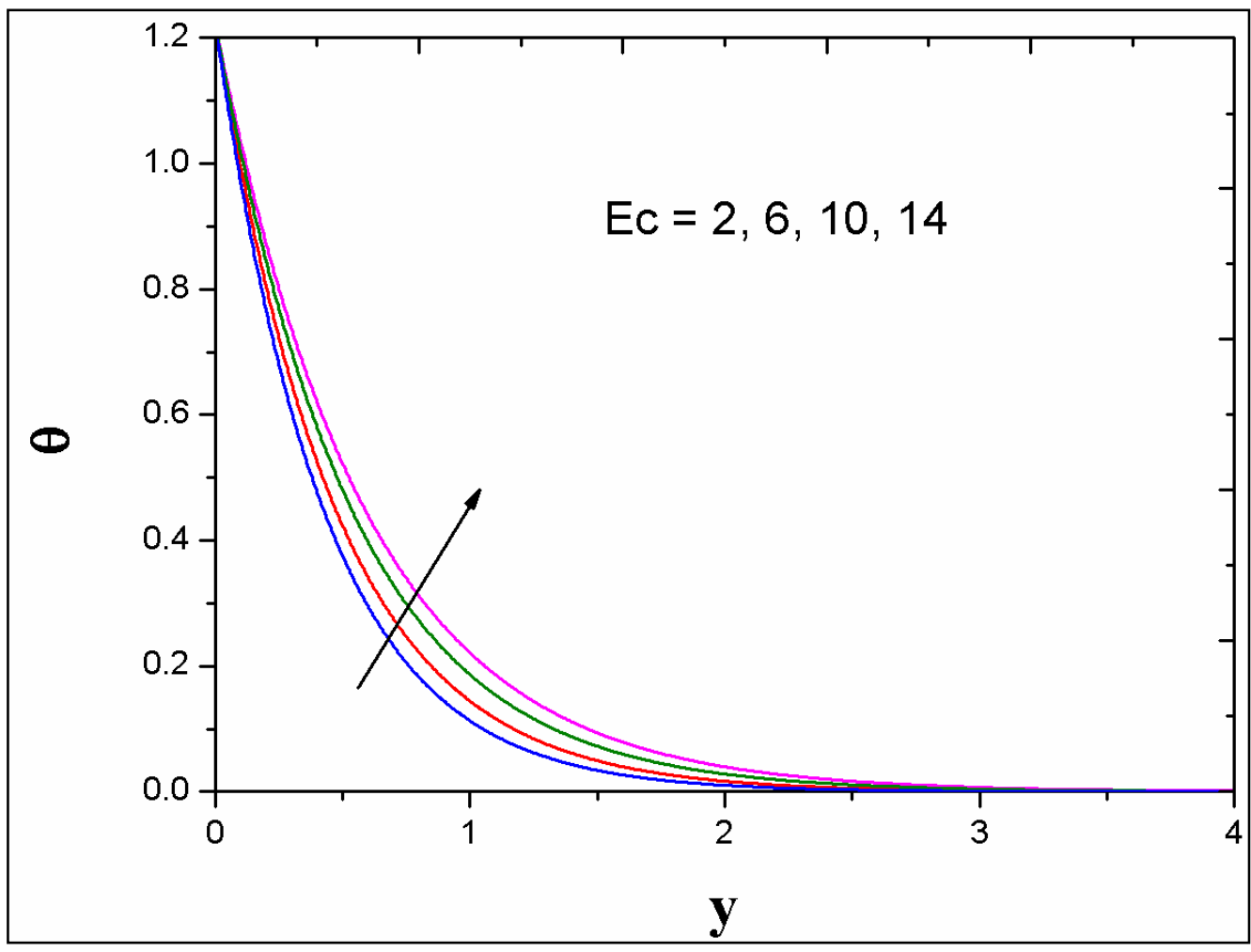

Figure 5: Temperature profile for different values of Eckert (viscous heating) number $(E c)$.

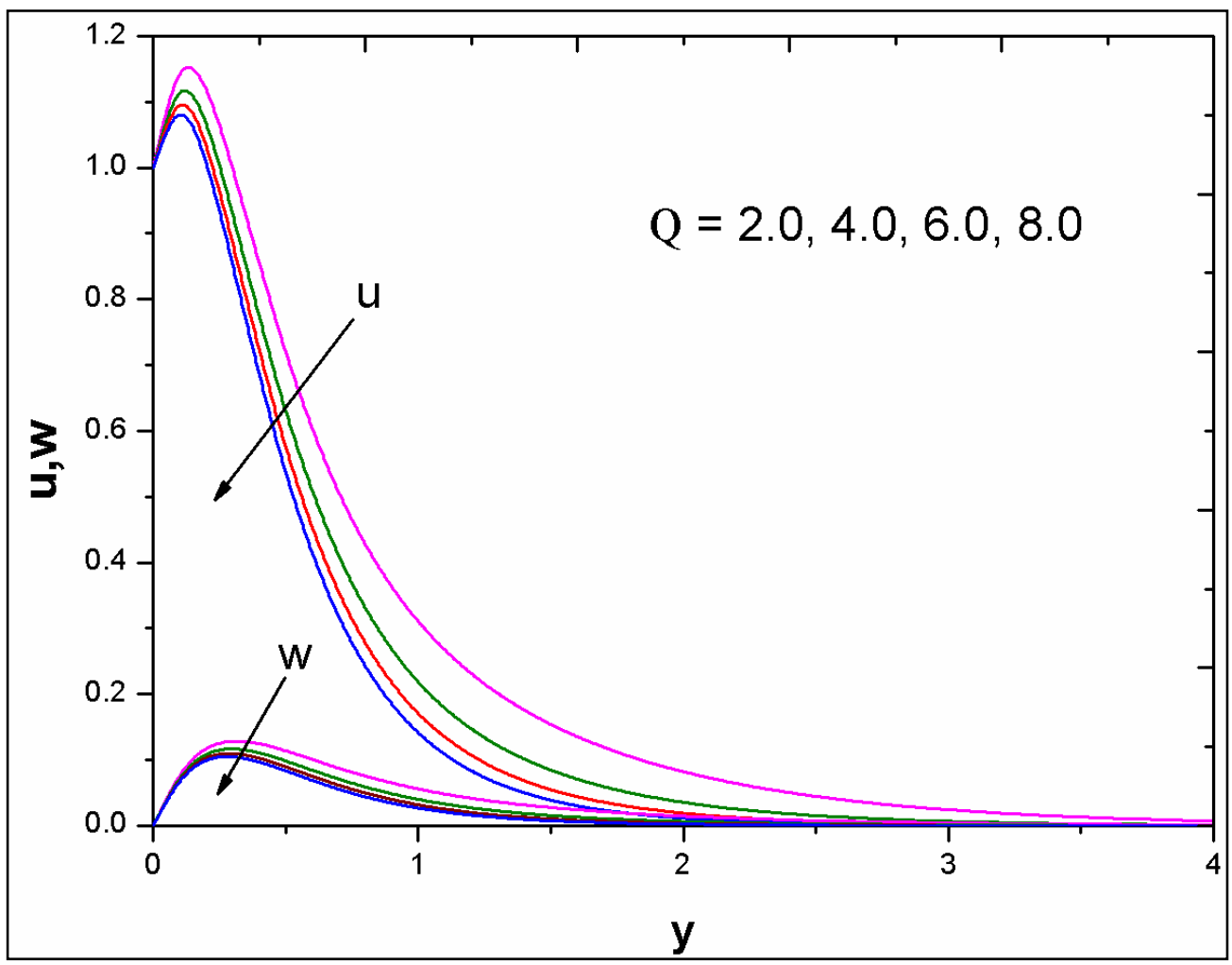

Figure 6: Primary and secondary velocity distributions for different heat source parameters $(Q)$ 


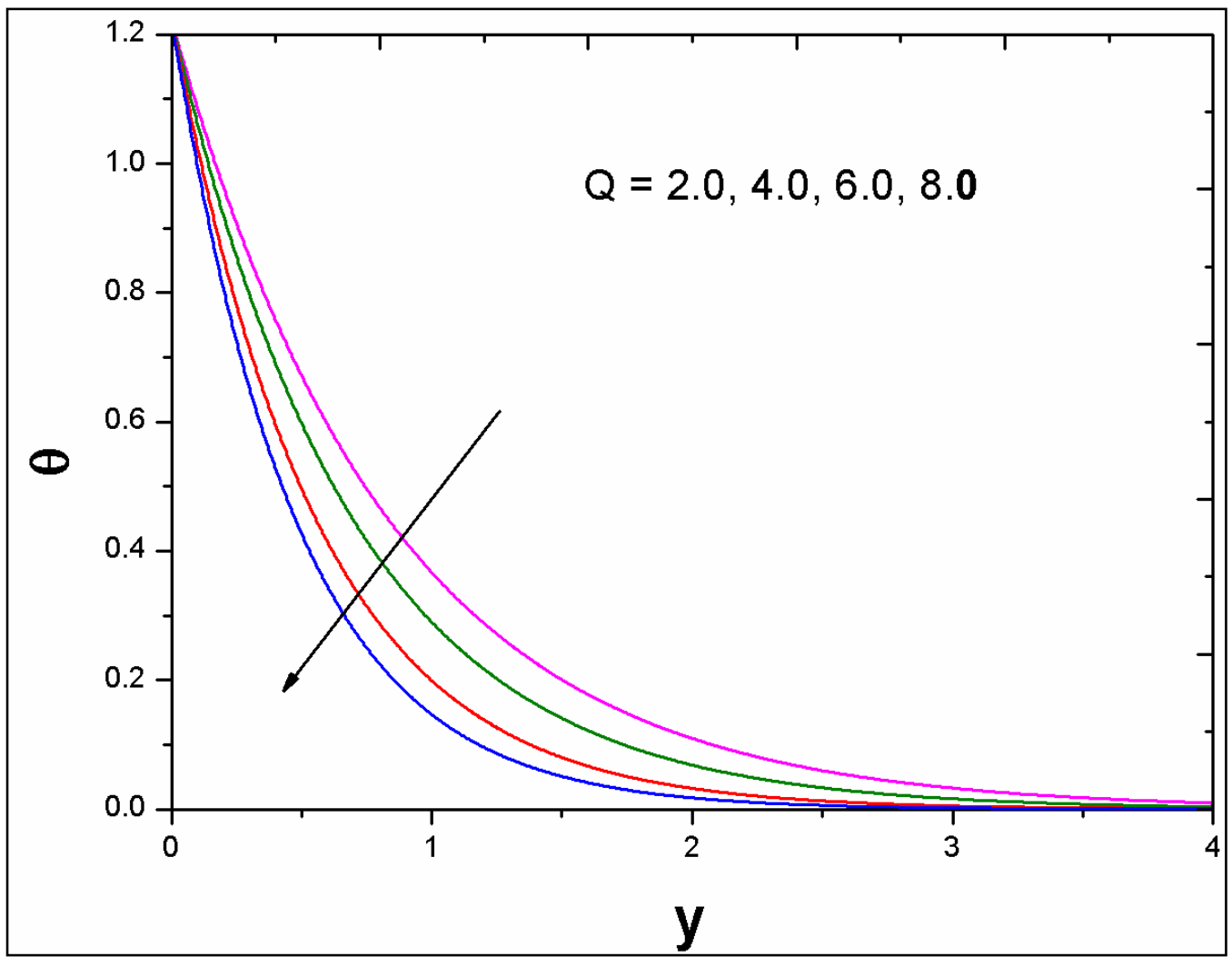

Figure 7: Temperature profile for different heat source parameters $(Q)$

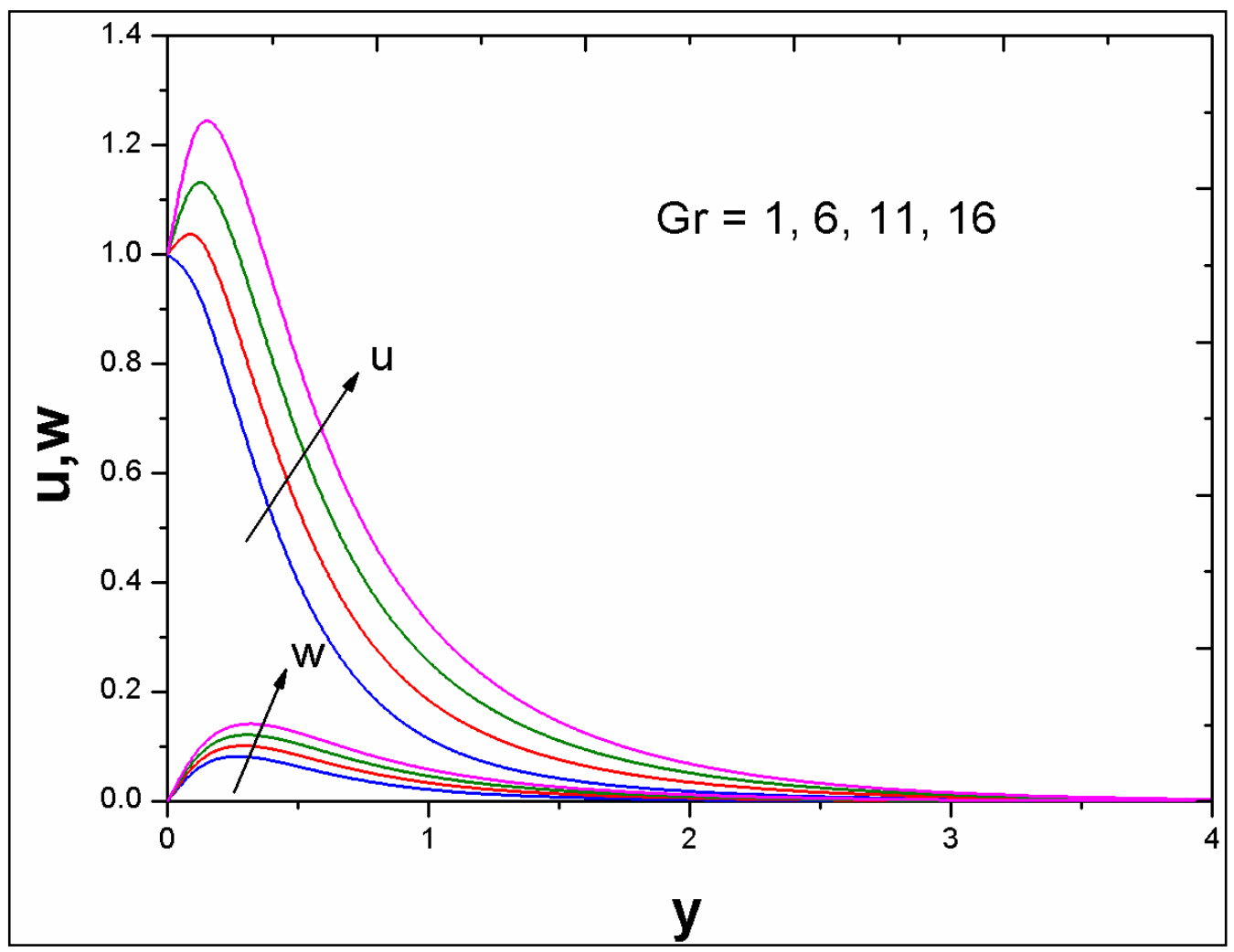

Figure 8: Primary and secondary velocity distributions for different thermal Grashof numbers $(\mathrm{Gr})$ 


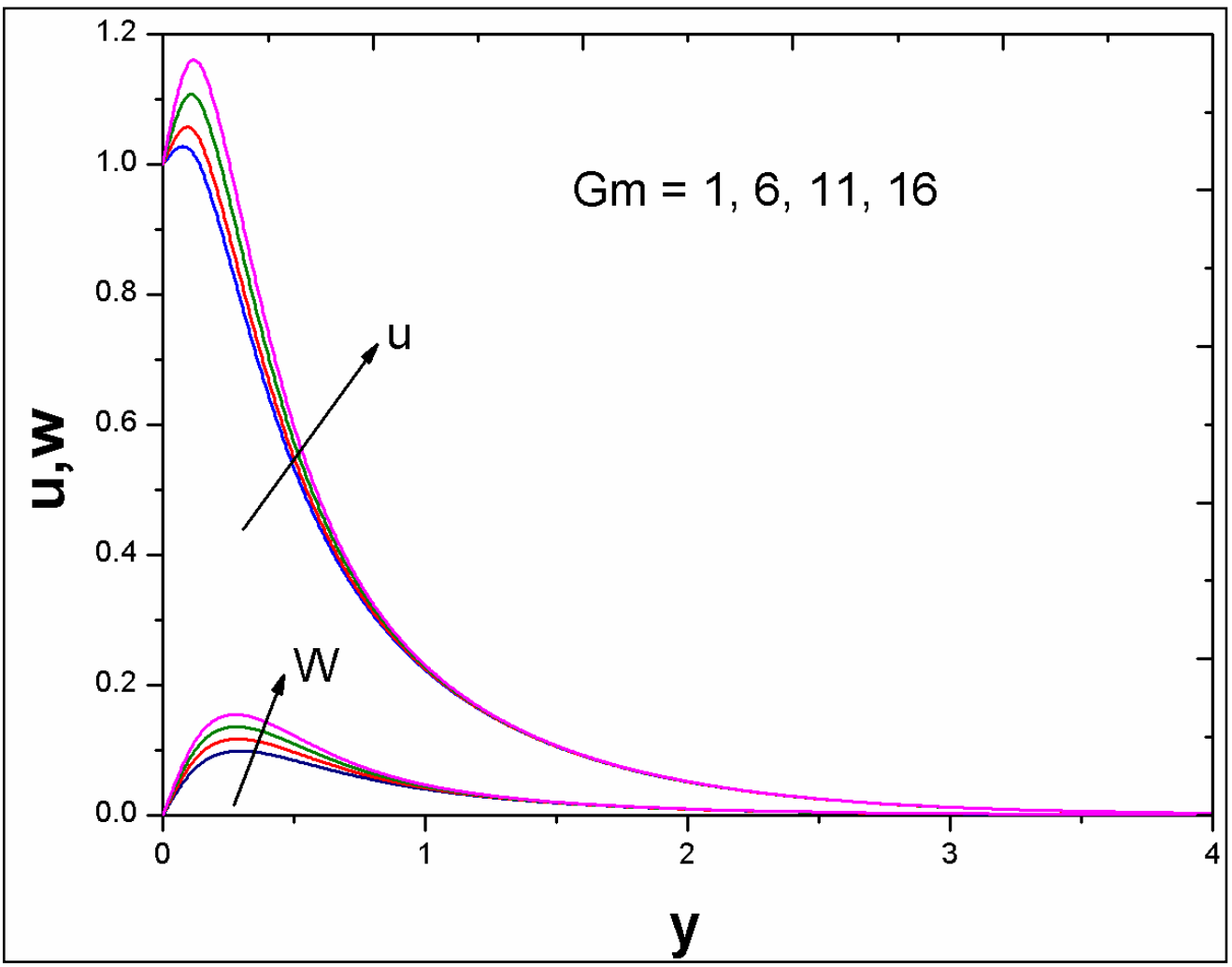

Figure 9: Primary and secondary velocity distributions for different species Grashof numbers $(\mathrm{Gm})$

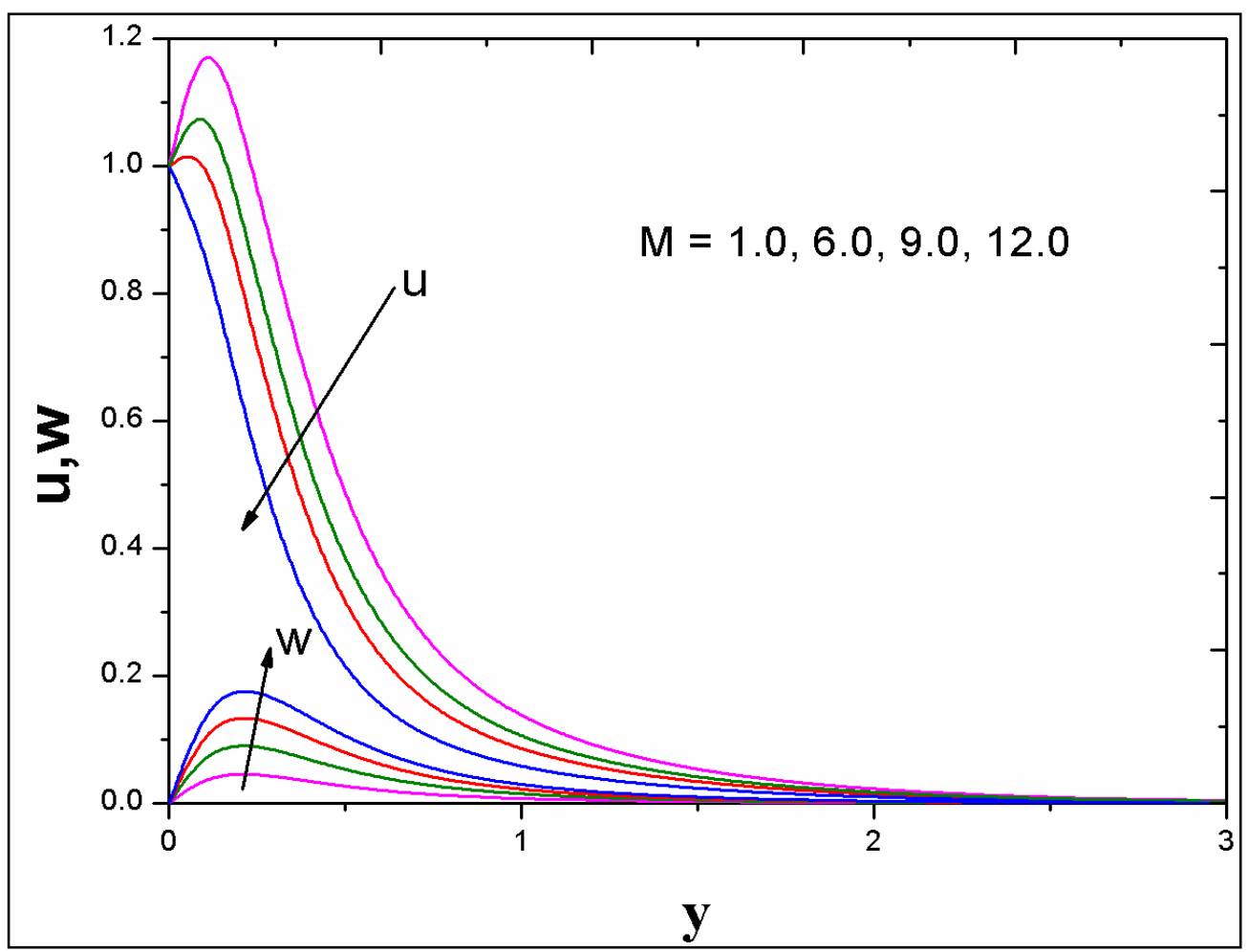

Figure 10: Primary and secondary velocity distributions for magnetic body force parameters $(M)$ 


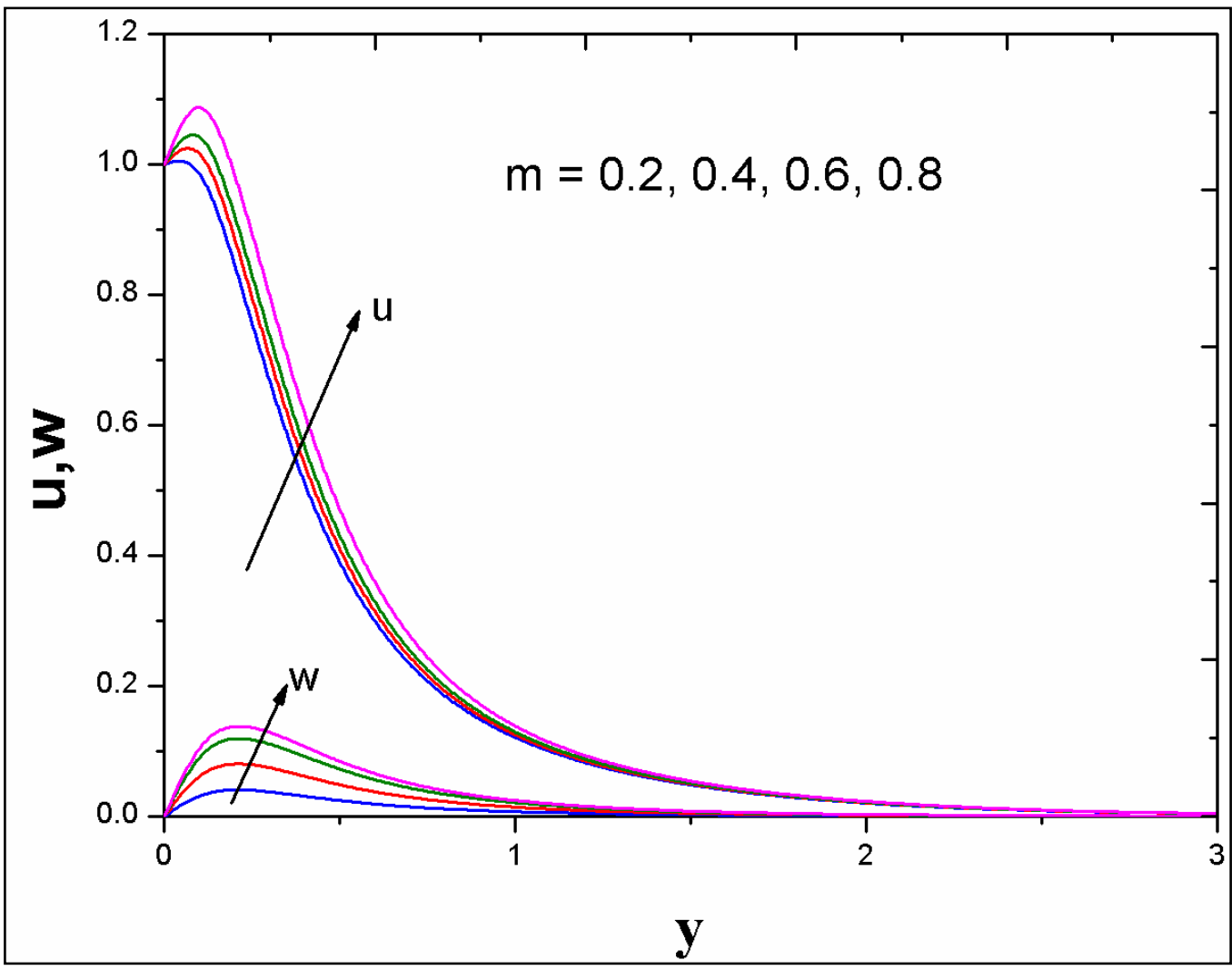

Figure 11: Primary and secondary velocity distributions for different Hall current parameters $(m)$

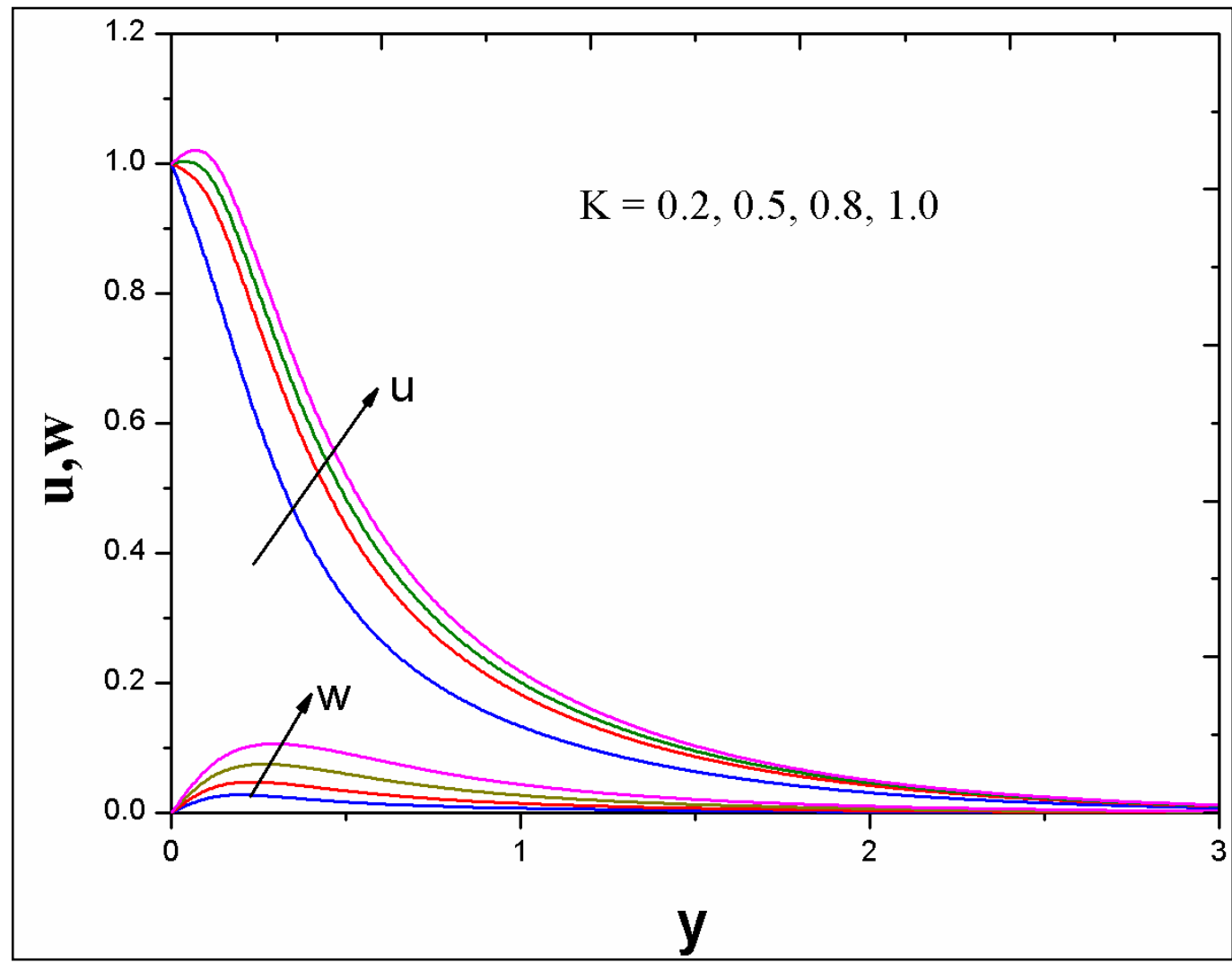

Figure 12: Primary and secondary velocity distributions for different permeability parameters $(K)$ 


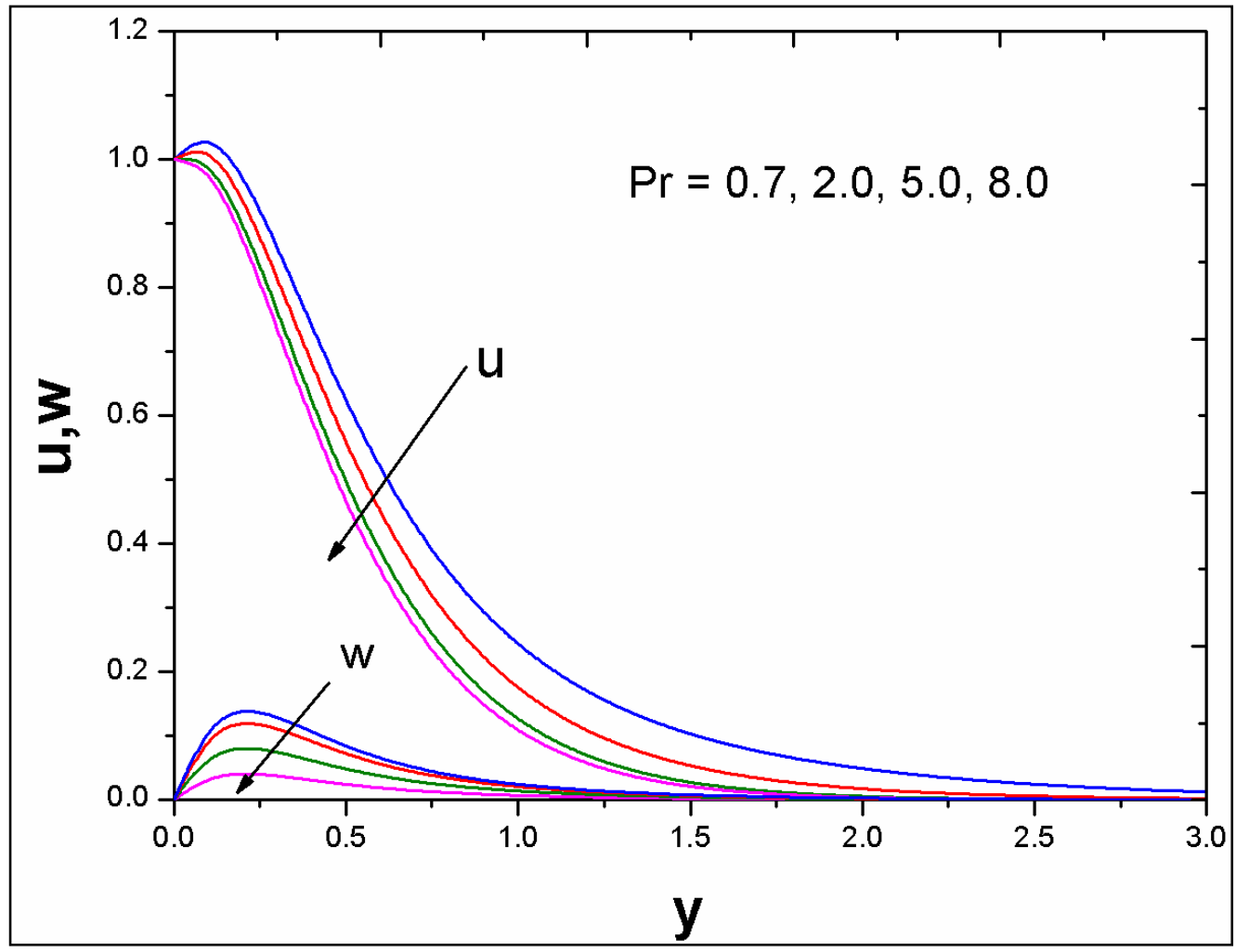

Figure 13: Primary and secondary velocity distributions for different values of Prandtl number (Pr)

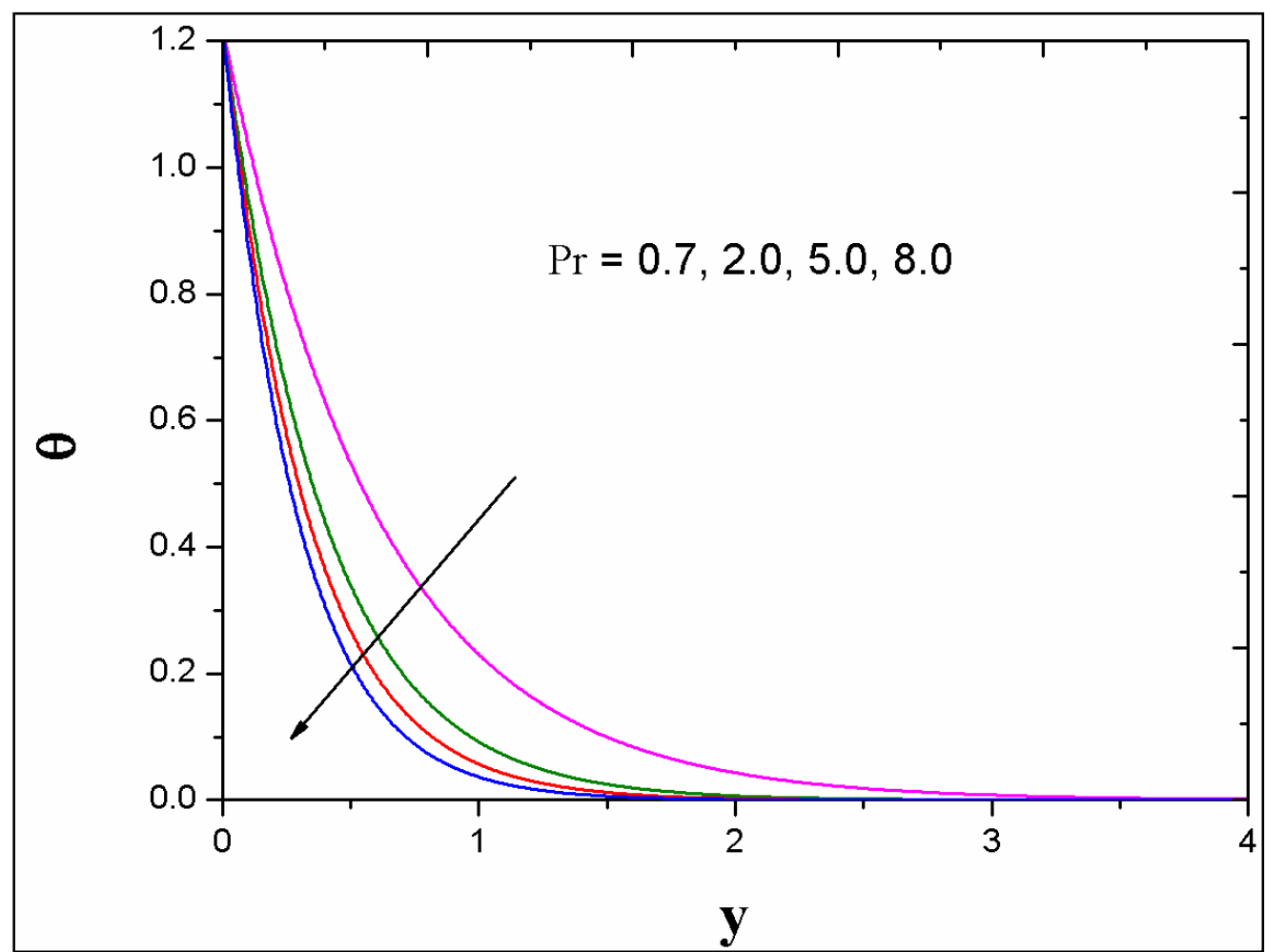

Figure 14: Temperature profile for different values of Prandtl number $(\mathrm{Pr})$ 


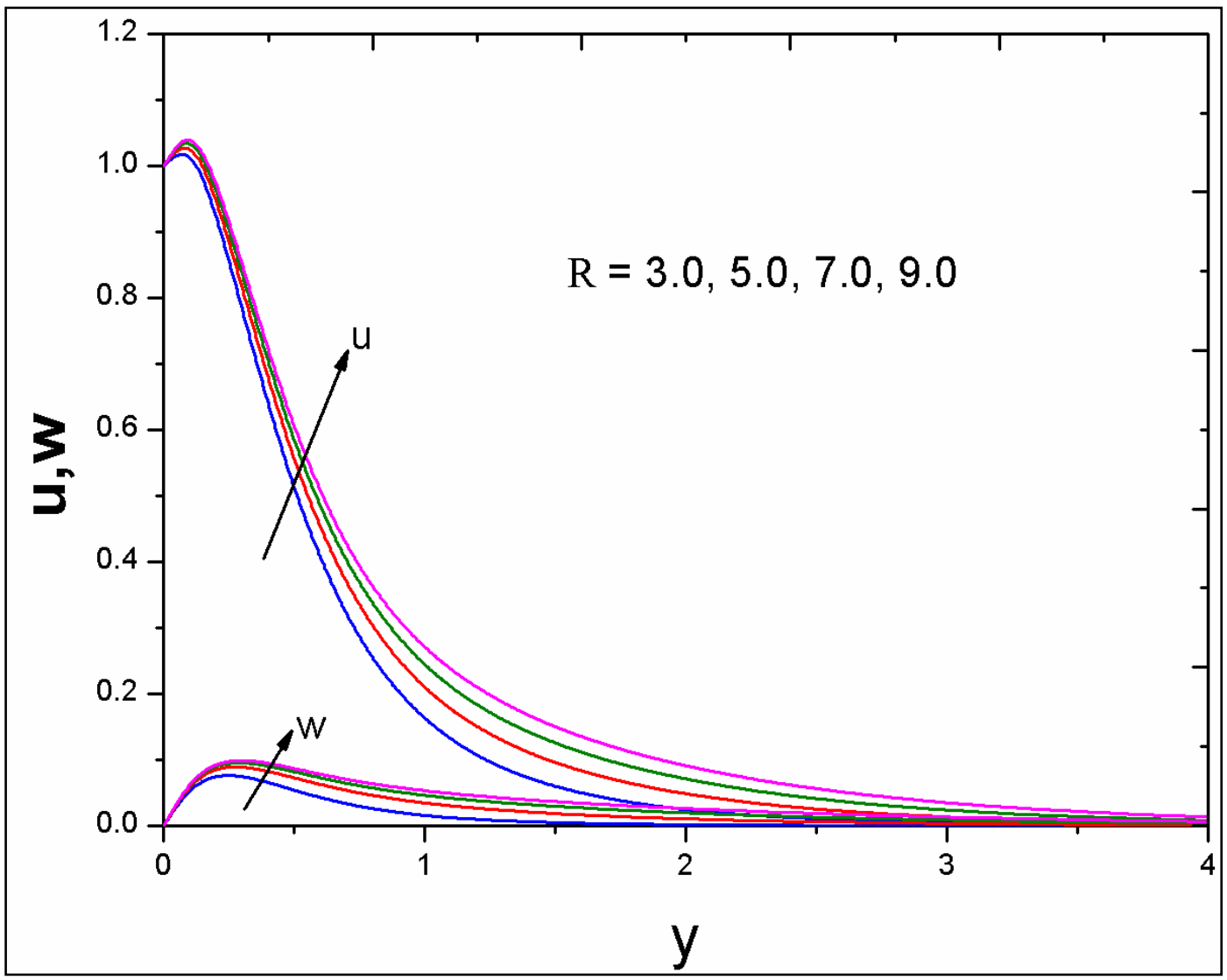

Figure 15: Primary, secondary velocity distributions for different radiation-conduction parameters $(R)$

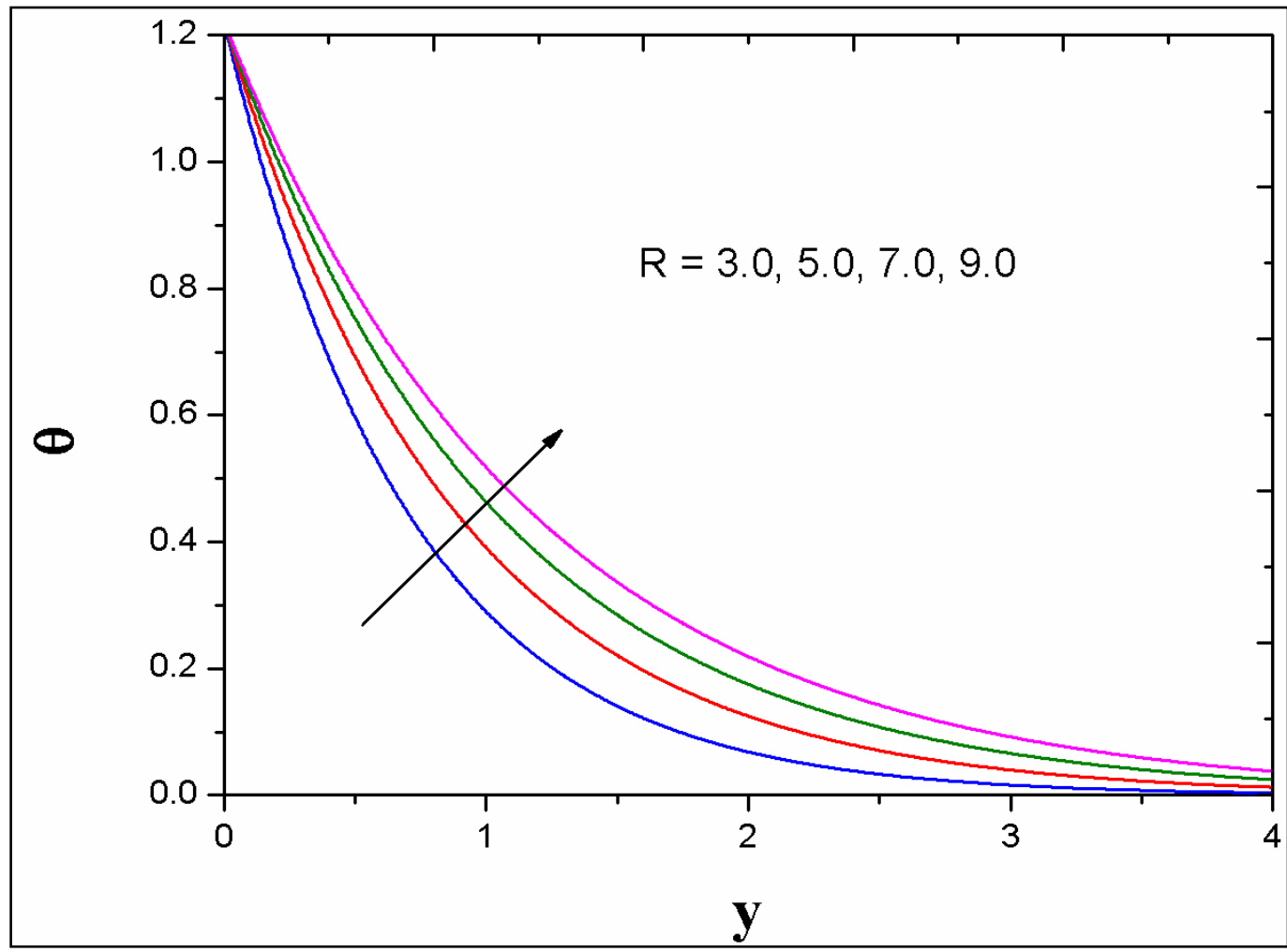

Figure 16: Temperature profile for different radiation-conduction parameters $(R)$ 


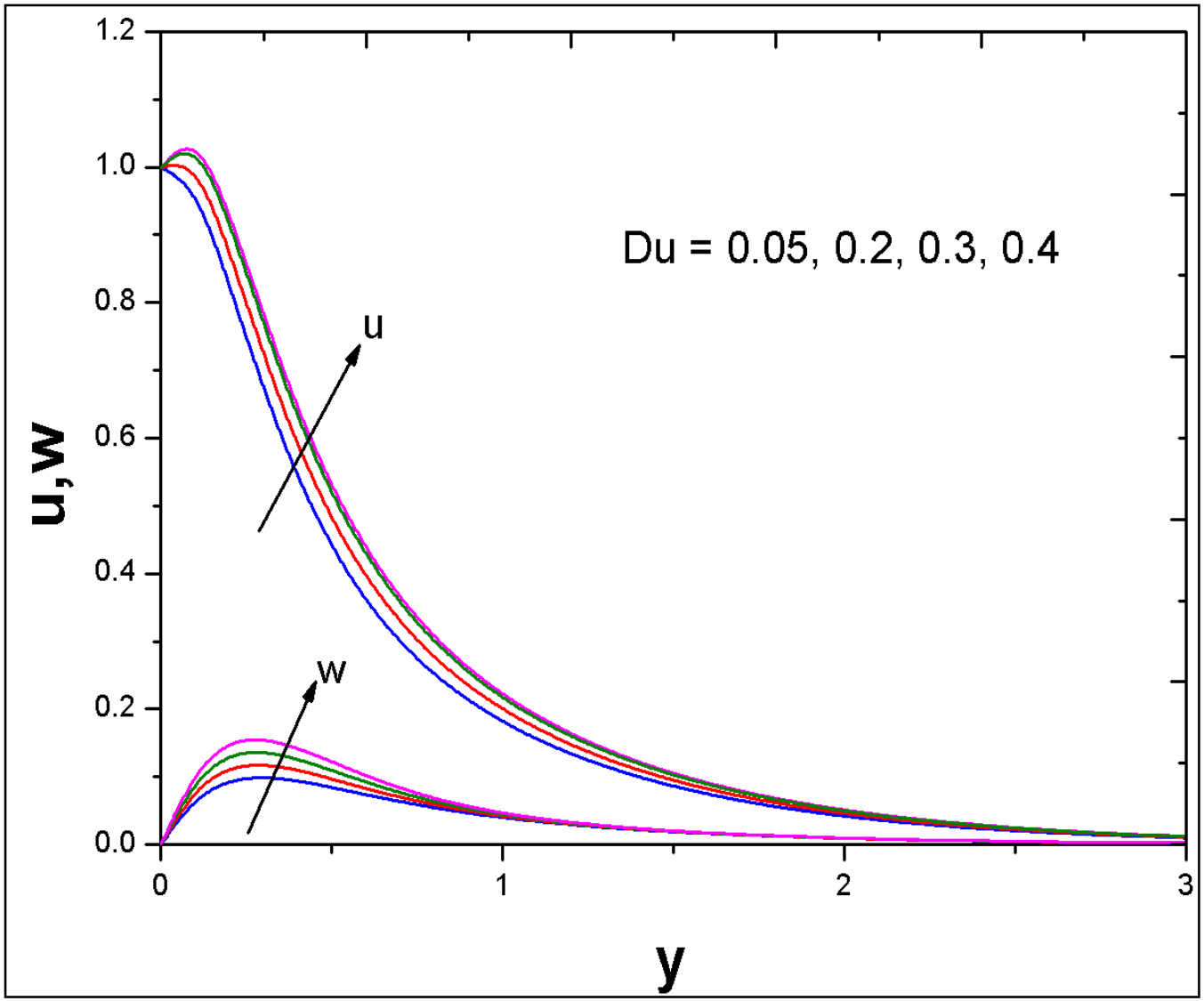

Figure 17: Primary and secondary velocity distributions for different Dufour numbers $(\mathrm{Du})$

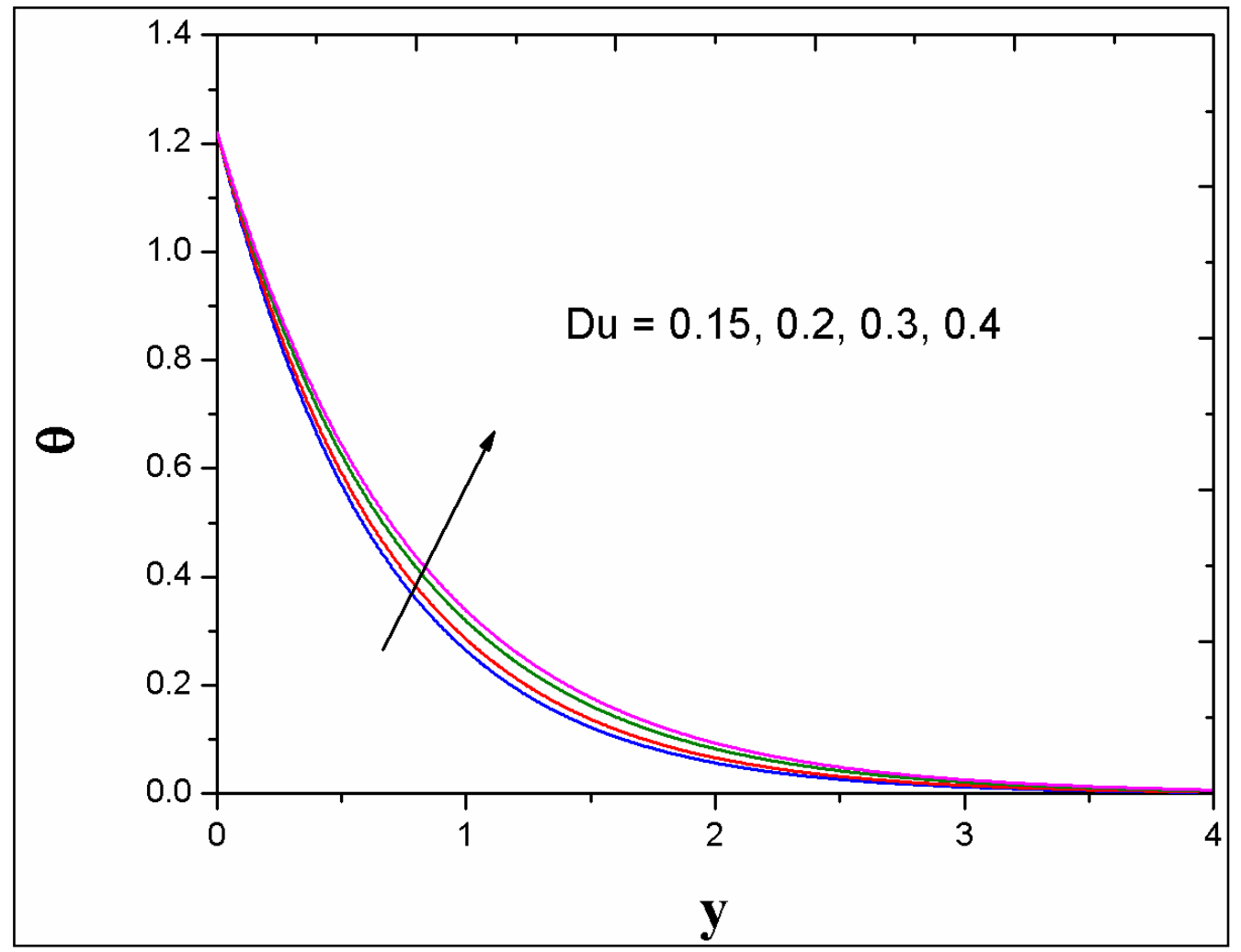

Figure 18: Temperature profile for different Dufour numbers $(D u)$ 


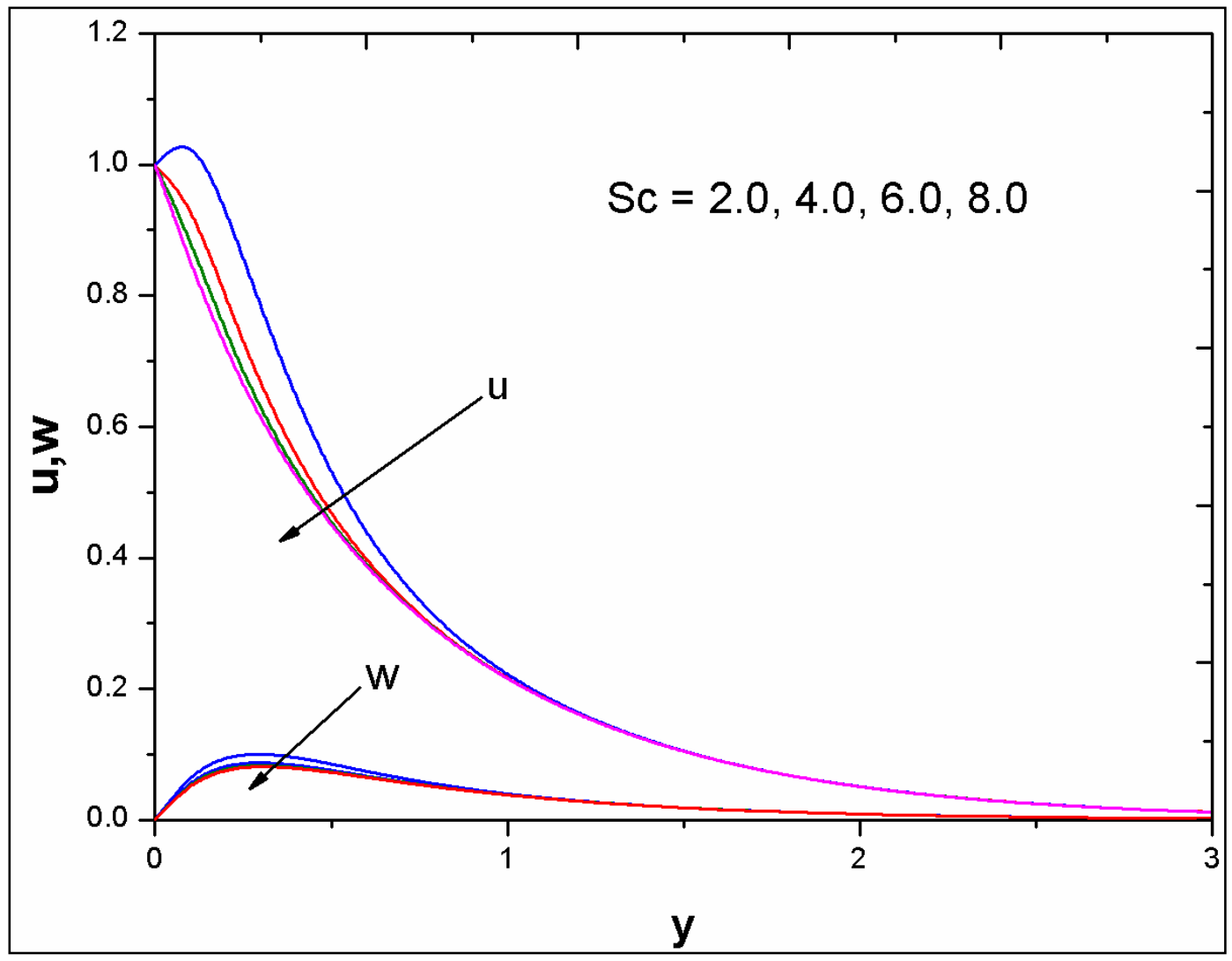

Figure 19: Primary and secondary velocity distributions for different Schmidt numbers $(S c)$

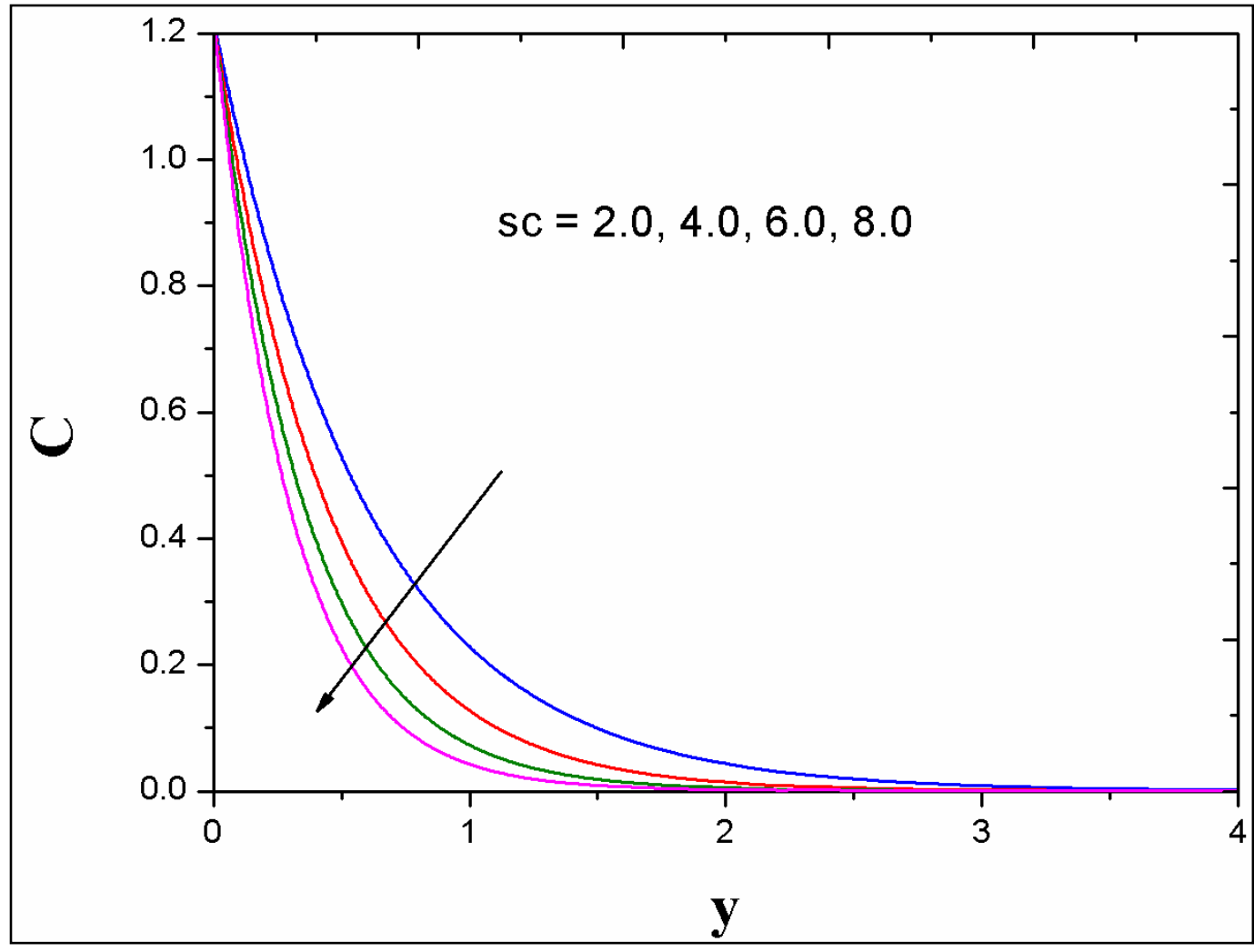

Figure 20: Concentration profile for different values of Schmidt number $(S c)$ 


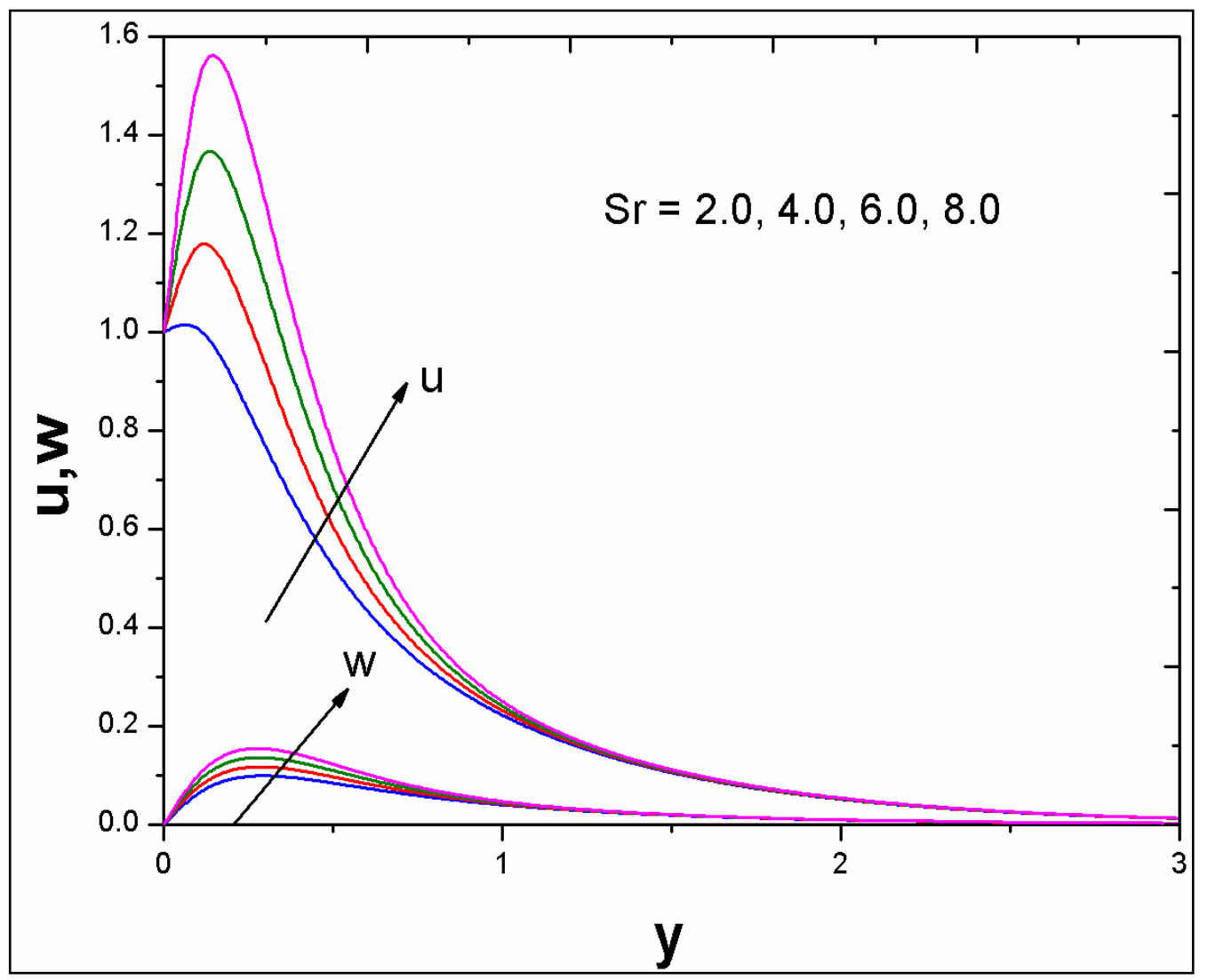

Figure 21: Primary and secondary velocity distributions for different values of Soret number $(\mathrm{Sr})$

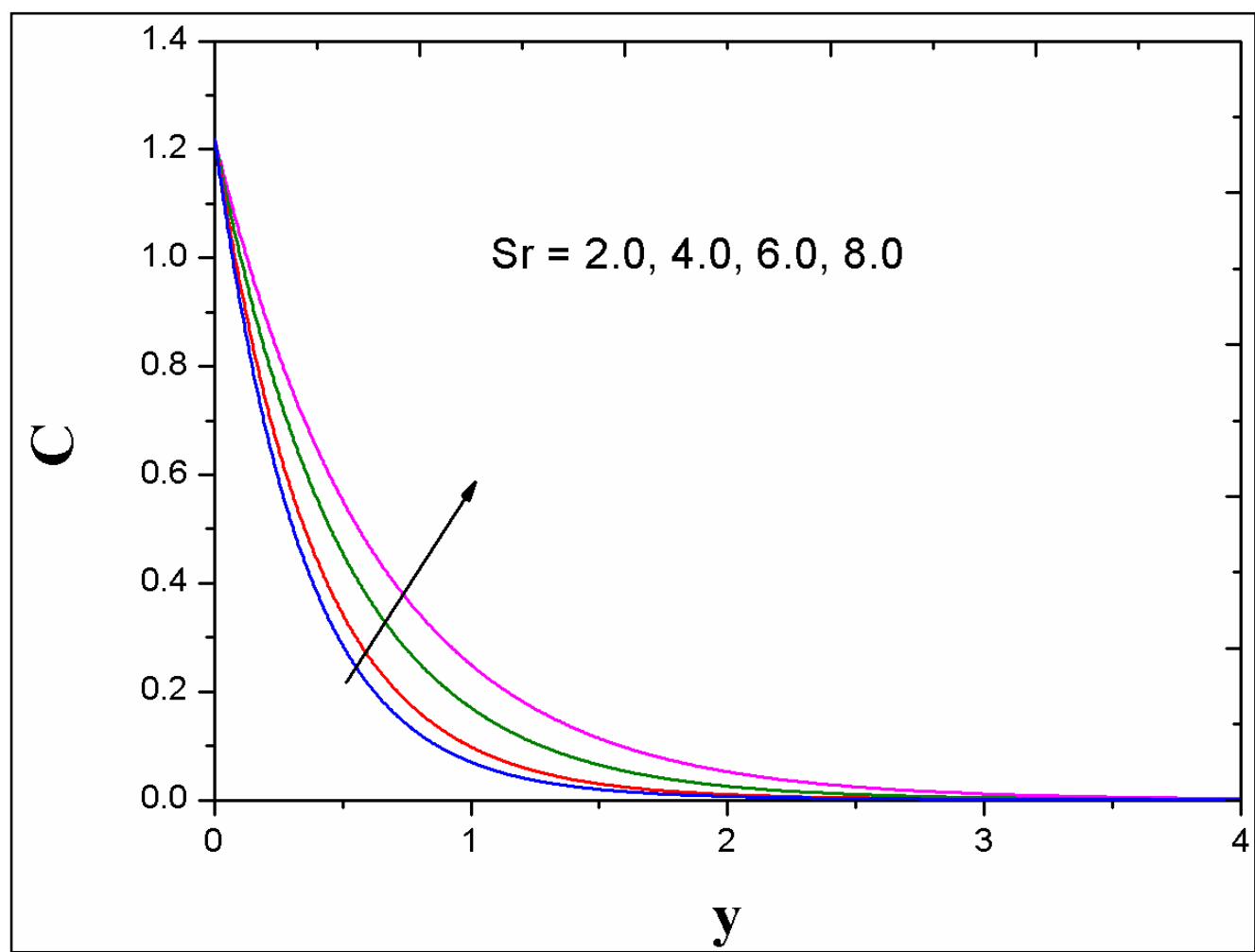

Figure 22: Concentration profile for different values of Soret number $(\mathrm{Sr})$ 


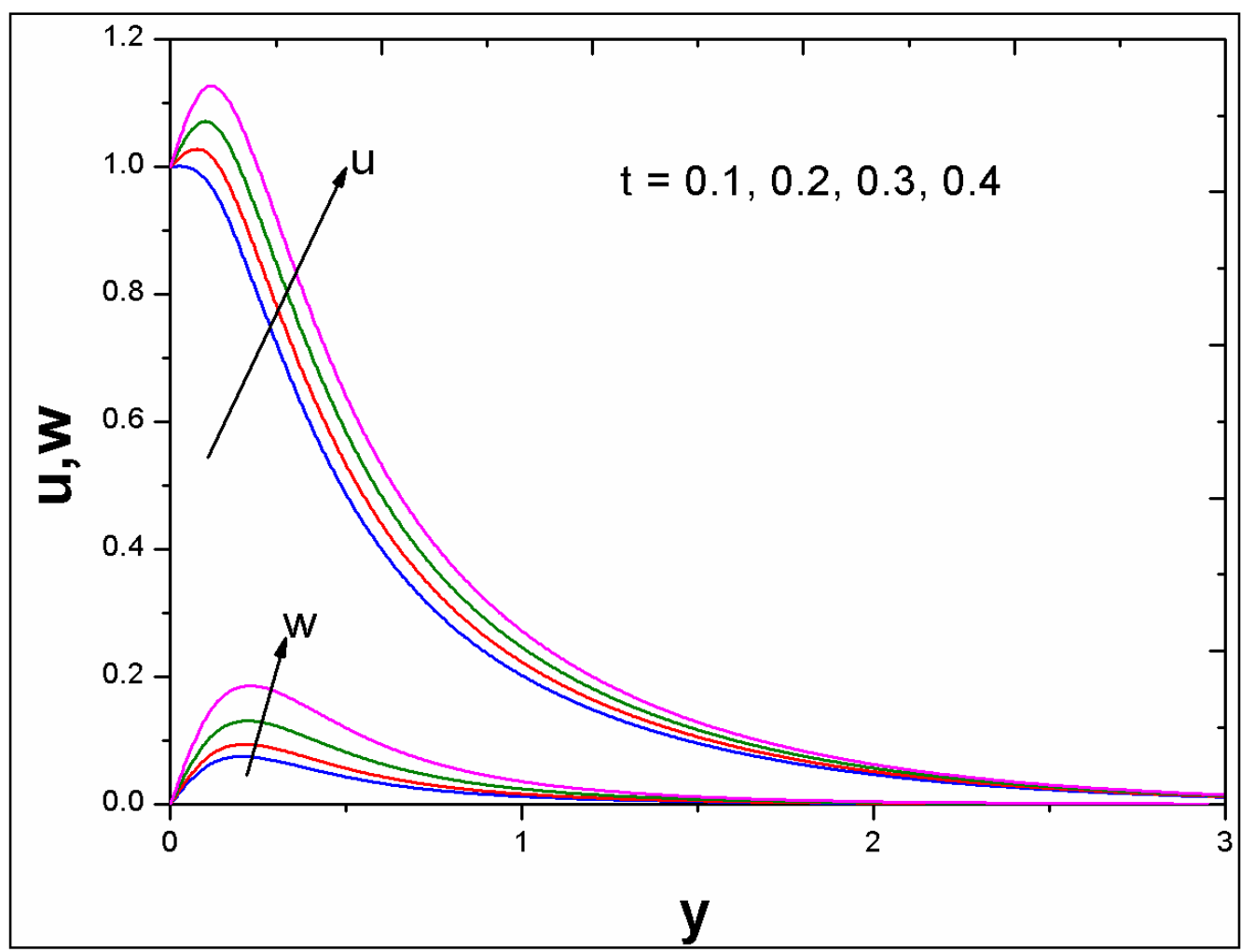

Figure 23: Velocity profile for different values of time $(t)$

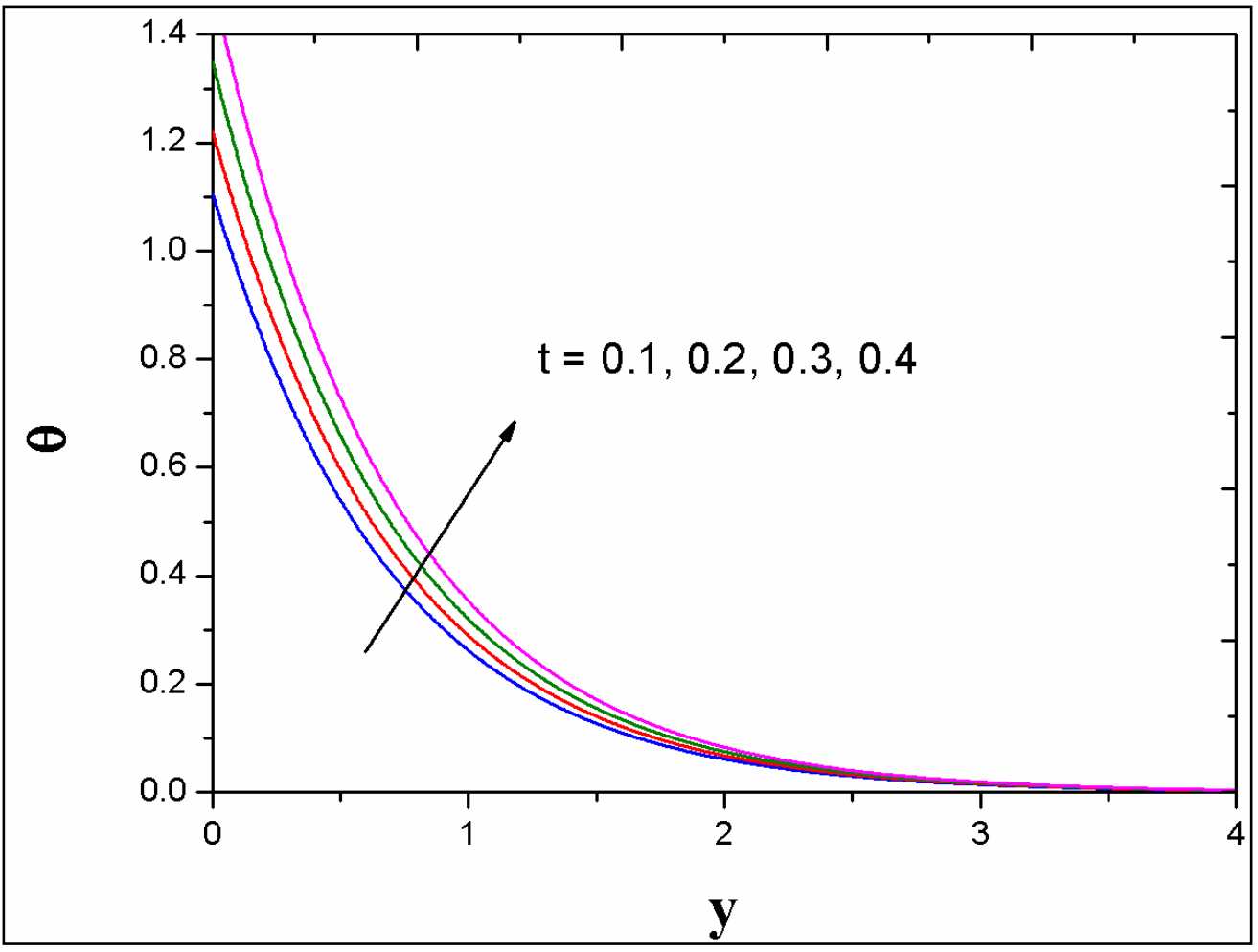

Figure 24: Temperature profile for different values of time $(t)$ 


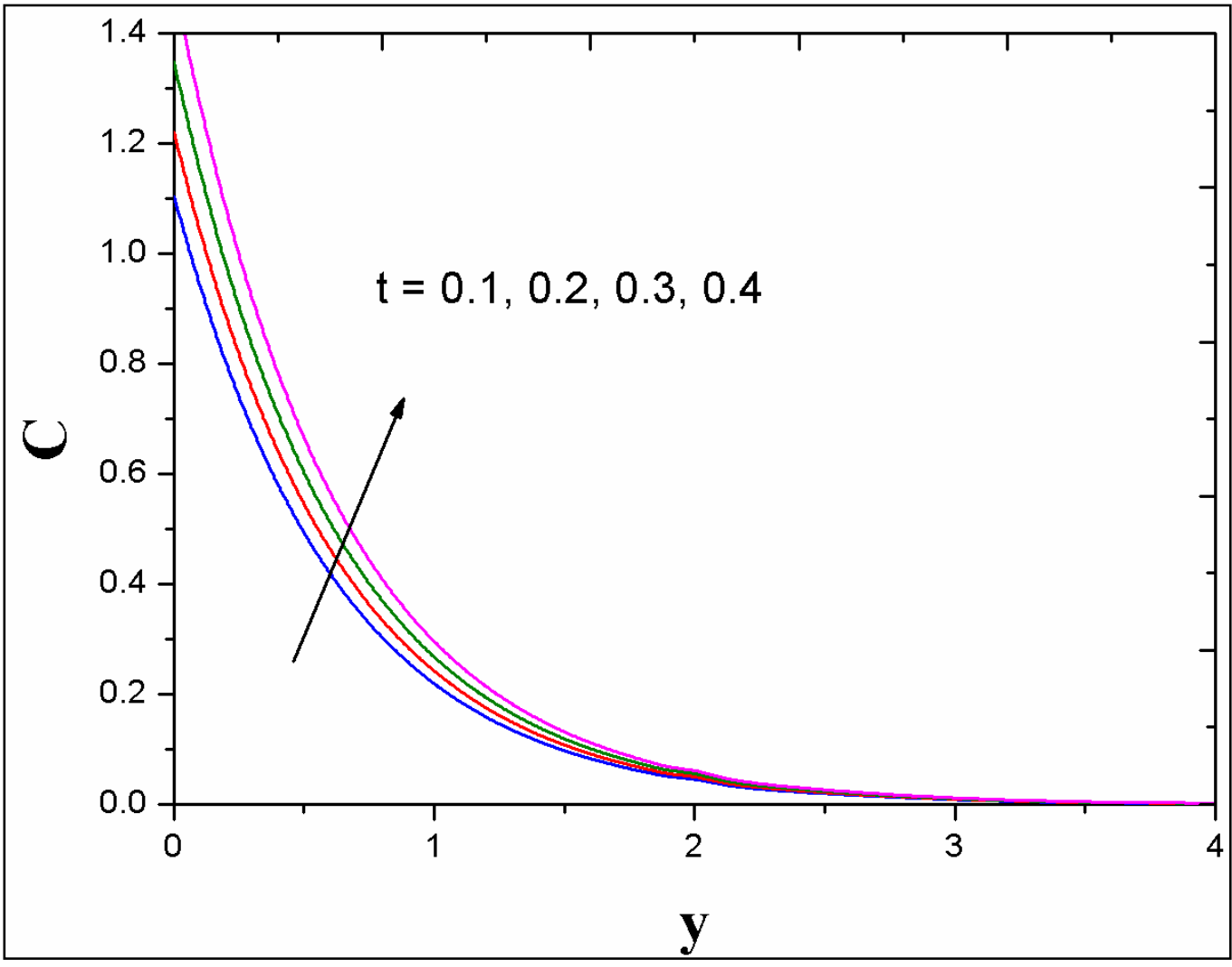

Figure 25: Concentration profile for different values of time $(t)$

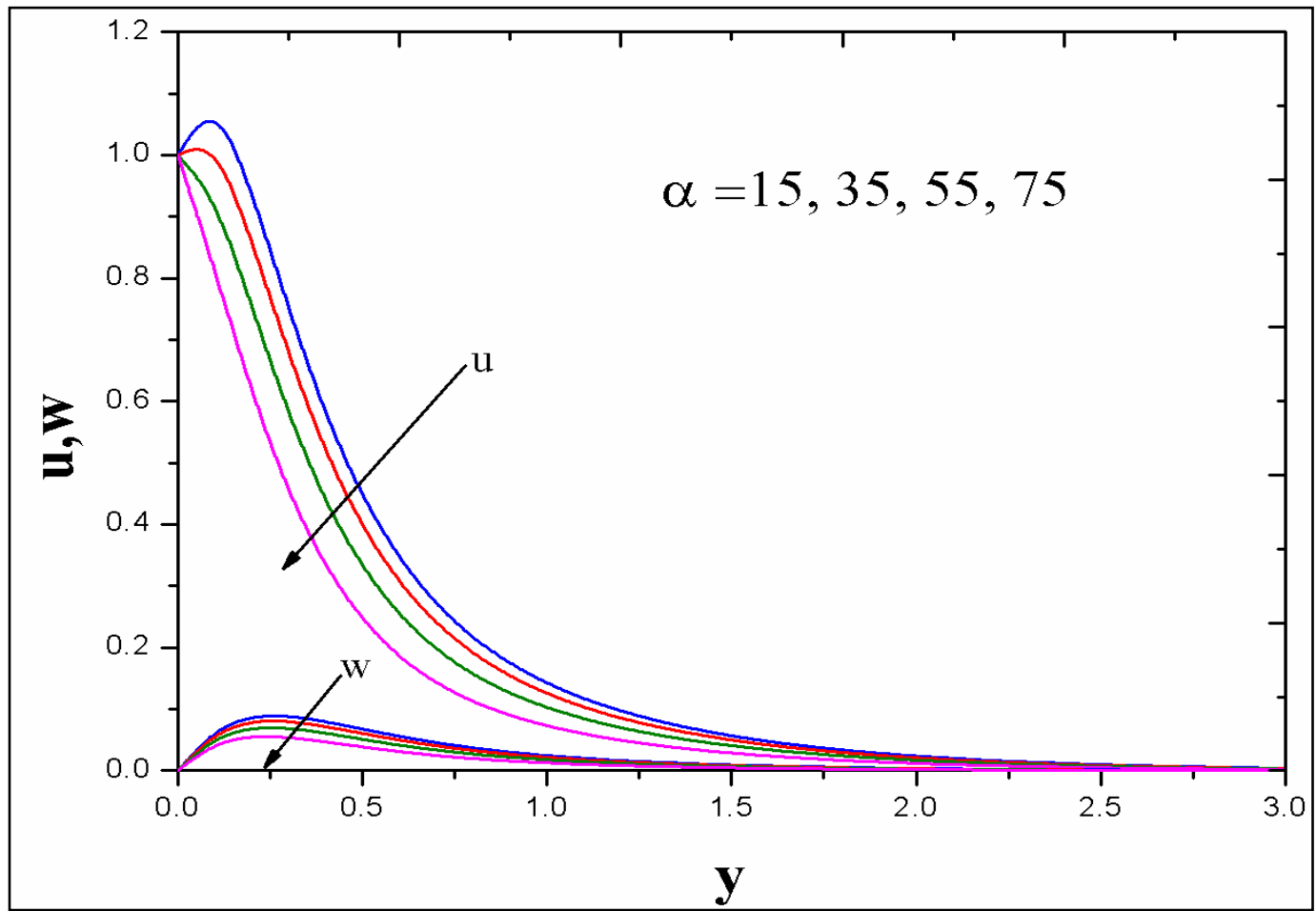

Figure 26: Velocity profile for different values of duct wall inclination $(\alpha)$ 
In all the profiles in Fig. 2 asymptotically smooth convergence of profiles in the free stream is computed indicating that an adequately large infinity boundary condition has been prescribed in the MATLAB FEM code. It is further noteworthy that even though primary and secondary deceleration is induced with greater strength of the chemical reaction, backflow is never induced nor is boundary-layer separation. Fig. 3 illustrates the response of the reactive species concentration to greater values of the chemical reaction parameter $(K r)$. A significant reduction in concentration magnitudes is observed which is consistent with the fact that in destructive homogenous chemical reactions, progressively more species will be converted via reaction. This will deplete molecular species levels and result in a thinning in the species boundary layer thickness. The presence of chemical reaction can be mitigated by anti-corrosion methods e.g. inhibitors or coatings and this has been considered in recent work by Kadir et al. [66]. However, it is intended that future studies by the authors will address in more detail the mechanisms entailed in surface corrosion in MHD generators which generally require more sophisticated computational fluid dynamics solvers and are therefore not tackled here.

Figures 4 and 5 illustrate the impact of the viscous dissipation parameter (Eckert number, Ec) on respectively primary and secondary velocity distributions and temperature. The Eckert number expresses the relative contribution of kinetic energy in the flow and the enthalpy difference in the boundary layer. At very low values this parameter embodies the conversion of kinetic energy into thermal energy by work done against the viscous fluid stresses. However, at higher values this dissipation effect can accelerate incompressible flows, especially when cross flow (Hall current) effect is present. Viscous fluid stresses have been shown to respond differently to dissipation effects (which occur at the molecular level) in magnetohydrodynamics, as noted by Rosa [67]. In the current quasi-three-dimensional flow regime, increasing $E c$ values serve to accentuate both the primary and secondary velocity and the associated boundary layer thicknesses are therefore decreased (Fig. 4). However, the influence is much more prominent in the primary flow field than the secondary flow field. Fig. 5 reveals that temperature increases with increase in viscous dissipation parameter, i.e. a rise in Eckert number elevates the thermal energy and heats the boundary layer. Positive Eckert number (as studied here) implies cooling of the wall and therefore a transfer of heat to the magnetized generator working fluid. The presence of a strong magnetic field $(M=2$ i.e. magnetic Lorentz force is twice the viscous hydrodynamic force) may also contribute to dissipation effects, as noted by Ibrahim and Shankar [68]. In the Hall MHD generator system, the effect of viscous dissipation is to energize the near-wall flow, yielding a greater fluid 
temperature which in conjunction with significant thermal and solutal (species) buoyancy forces increases thermal boundary layer thickness. The implication is that in mathematical models of Hall current generator flow, which neglect viscous heating, temperatures will be under-predicted and inaccurate determinations of heat transfer rates at the duct wall will be computed. It is therefore important in realistic simulation of MHD generators to include the viscous dissipation effect.

Figures 6 and 7 displays the effect of heat absorption parameter $(Q)$ on the primary and velocity fields and temperature distribution, respectively. The case $Q>0$ corresponds to a heat sink and $Q<0$ to a heat source (generation). Hot and cold spots may arise in MHD generators depending on the length of the duct [67]. the presence of heat absorption (thermal sink) deenergizes the flow and reduces the fluid temperature (fig. 7). This inhibits thermal buoyancy (and also species buoyancy, via coupling) and manifests in a net deceleration in both the primary and secondary velocity components (fig. 6). Effectively the presence of a heat sink will generate an increase in both primary and secondary momentum boundary layer thicknesses whereas it will reduce the thermal boundary layer thicknesses.

Figures 8 and 9 depict the effect of thermal Grashof number ( $G r$ ) and solutal Grashof number $(G m)$ on the primary and secondary velocity distributions $(u, w)$. Each of these numbers exert a significant influence via respectively the thermal buoyancy force term $(+G r \cos \alpha . \theta)$ and species buoyancy force $(+G m \cos \alpha . C)$ which appear in the primary momentum Eqn. (12) only. The thermal Grashof number ( $G r$ )signifies the relative magnitude of the thermal buoyancy force to the viscous hydrodynamic force in the boundary layer. For $G r=1$ both forces contribute equally whereas for $G r>1$ thermal buoyancy dominates the viscous force. As expected, it is observed that there is accentuation in both primary and secondary velocity (fig. 8) i.e. dual acceleration is instigated, with increasing $G r$ values. Primary and secondary boundary layer thicknesses are therefore reduced with enhancement of thermal buoyancy force. It is important to note that MHD generator flows are not restricted to forced convection; indeed, with inclined duct features, buoyancy effects can be greatly exploited as noted by Rosa [67], Aboeldahab and Elbarbary [27] and Bég et al. [35]. Positive values of Gr correspond to cooling of the duct wall (plate) by free convection currents. The case of negative $G r$ is not considered as this creates the opposite effect and heats the wall which is undesirable with chemical corrosion effects. Substantially higher primary velocity magnitudes are obtained compared with secondary velocity. Overshoots near the wall (characteristic of MHD boundary layers) are also much sharper in the primary flow than in the secondary flow. Additionally, there is a much 
greater dispersion in primary velocity profiles over the same increment in thermal Grashof number compared with the secondary velocity profiles which are much more clustered together. In both primary and secondary velocity distributions there is evidently a smooth decay into the free stream indicating again that a sufficiently large infinity boundary condition has been implemented in the MATLAB FEM code. The solutal Grashof number $(\mathrm{Gm})$ defines the ratio of the species buoyancy force to the viscous hydrodynamic force and is used to characterize the buoyancy effects generated by the molecular reactive species diffusing in the magnetic fluid. A similar response is generated as with the thermal buoyancy effect i.e. both primary and secondary velocity are elevated with increasing $G m$ values (fig. 9). Although smaller magnitudes of velocity components are computed relative to fig. 8 , there is a sharper topology to the primary velocity overshoots which are displaced closer to the duct wall. Monotonic decays to the free stream are again observed with progressive distance from the duct wall surface.

Figure 10 displays the velocity profiles $u, w$ for various values of magnetic body parameter $M$.In Hall current flows the conventional Lorentz body force term is augmented by Hall parameter. The collective modified body force terms for the primary and secondary flow feature in both the primary momentum equation and the secondary momentum equation, and respectively, take the form:

$$
\begin{gathered}
\left.-M / 1+m^{2}\right) u-\left(m M / 1+m^{2}\right) w \\
\left.-\left(M / 1+m^{2}\right) w+m M / 1+m^{2}\right) u \ldots .
\end{gathered}
$$

Evidently both body forces are negative in the primary momentum Eqn (28) whereas only the secondary contribution is negative in the secondary momentum Eqn (29). It is observed that the primary velocity profile decreases whereas the secondary velocity profile increases with elevation in the magnetic field parameter. The primary momentum is inhibited by Lorentzian drag whereas the secondary momentum is augmented owing to the cross-flow re-distribution of momentum in the regime, a feature which has been observed in a number of investigations including Rosa [67], Cramer and Pai [60] and Stankiewicx and Palmer [14]. The presence of Hall current therefore substantially alters the impact of Lorentzian magnetic body force on the fluid dynamics.

Figure 11 depicts the velocity components for different values of the Hall parameter $(m)$. A more consistent response is observed in this case. Both primary and secondary flows are 
assisted strongly with increasing Hall parameter. The cross-flow effect therefore benefits the primary flow also and results in a thinning in both primary and secondary boundary layer thicknesses.

Figure 12 visualizes the evolution in primary and secondary velocity profiles with permeability of the porous medium $(K)$ As noted earlier the Darcy model is employed in the simulations which is valid for low speed transport. Substantial enhancement in both primary and secondary velocity components is observed with an increase in permeability of porous medium. The Darcian impedances are respectively $-u / K$ and $-w / K$ in the primary momentum Eqn. (12) and secondary momentum Eqn. (13). Increasing $K$ values correspond to a depletion in resistance of the solid fibers to the percolating magnetic fluid since hydraulic conductivity increases with lesser and lesser solid material. This accelerates both primary and secondary flows since the Darcian drag forces are reduced (inverse proportionality with permeability). Maximum primary velocity and secondary velocity are therefore computed with the highest permeability parameter value and the minimal Darcian retarding force. As $K \rightarrow \infty$, the porous matrix will disappear. The regime will therefore resort to purely viscous magnetic fluid. In the opposite limit as $K \rightarrow 0$, the medium permeability will vanish and the MHD generator duct space will be completely filled with solid material preventing operations.

Figs 13 and 14 depict the primary and secondary velocity $(u, w)$ profile evolution and temperature profile $(\theta)$ across the boundary layer for different values of the Prandtl number, $\operatorname{Pr}$. It is seen that an increase in Pr leads to a decrease in both primary and secondary velocity magnitudes (fig. 13). Fig. 14 shows that an increase in the Prandtl number Pr results in a decrease in temperature and also the thermal boundary layer thickness and a more uniform temperature distribution across the boundary layer. The reason is that smaller values of Pr are equivalent to an increasing thermal conductivity, and therefore heat is able to diffuse away from the heated surface more rapidly than at higher values of Pr. Hence the boundary layer becomes thicker and the rate of heat transfer is reduced. The thermal boundary layer thickness is the distance from the body at which the temperature is $99 \%$ of the temperature found from an inviscid solution. The ratio of the two thicknesses is dictated by the Prandtl number. For Prandtl number of unity, both the hydrodynamic and thermal boundary layers are of the same thickness. However, when Prandtl number exceeds unity, the thermal boundary layer is thinner than the velocity boundary layer whereas the converse is the case when Prandtl number is less than unity. Generally, higher $\operatorname{Pr}$ fluids will have relatively low thermal conductivities which 
will suppress thermal conduction heat transfer from the wall and reduce thermal boundary layer thickness, resulting in lower temperatures in the boundary layer regime.

Figures 15 and 16 illustrate the evolution in primary and secondary velocity and temperature with the radiation-conduction parameter $(R)$, respectively. Significant enhancement in both primary and secondary velocity (fig. 15) is induced with higher values of $R$. A substantial elevation is also caused in temperature (fig. 16). Both primary and secondary momentum boundary layer thicknesses are therefore reduced whereas thermal boundary layer thickness is elevated with increasing radiation-conduction parameter. Radiative heat transfer is dominant when the radiant heat flux, is large compared to the heat transfer rate due to convection or conduction. Typically, this will occur under high-temperature conditions, associated with MHD power generation [67] for which the fourth-order dependence of the radiative heat flux on temperature implies that the radiation heat transfer will dominate. The parameter, $R=$ $\frac{4 \sigma T t_{\infty}^{3}}{k m k}$, quantifies the importance of conduction versus radiation within the fluid. The parameter $R$ arises in the augmented energy Eqn. (14) i.e. in the term $\frac{1}{P r} \cdot\left(1+\frac{4}{3} \mathrm{R}\right) \quad \partial^{2} \theta / \partial y^{2}$. Rosseland's model assumes radiative equilibrium and that the fluid has gray properties which are physically viable in MHD generator operations under uni-directional radiative thermal loading [67]. Furthermore, Rosseland's model assumes that the intensity is the black-body intensity at the fluid temperature and since it is generally confined to incompressible flows it is particularly appropriate for viscous-dominated transport. Conductive heat transfer dominance is ensured in the thermal boundary layer, when $R \rightarrow 0$ i.e. the radiative contribution vanishes. With $R>0$ thermal radiation influences the regime i.e. a progressively greater quantity of radiation heat transfer is received within the boundary layer along the duct wall which results in energization of the fluid and a rise in temperatures. This behaviour is indeed captured in Fig. 16. The energization of the flow regime will serve to enhance thermal diffusion and therefore elevates temperatures and also thermal boundary layer thickness.

Figure 17 and 18 exhibit the velocity and temperature profiles for different values of Dufour number $D u$. The Dufour number $D u$ denotes the contribution of the concentration gradient, $\partial^{2} \mathrm{C} / \partial \mathrm{y}^{2}$. to the thermal energy flux in the flow. It can be seen that an increase in the Dufour number causes a rise in both primary and secondary velocity (fig. 17) and temperature (fig. 18) throughout the boundary layer. Concentration gradients, via coupling between the 
energy eqn. (14) and species eqn. (15) clearly reduce primary and secondary hydrodynamic boundary layer thicknesses whereas they elevate thermal boundary layer thickness.

Figures 19 and 20 illustrate the variation in primary, secondary velocity and temperature profile with various Schmidt numbers, Sc. Strong reduction in both primary and secondary velocity is induced with an increase in Schmidt number (fig. 19). Similarly, a significant decrease in temperature is generated with a rise in Schmidt number (fig. 20). The Schmidt number embodies the ratio of the momentum to the mass (species) diffusivity. It therefore quantifies the relative effectiveness of momentum and mass transport by diffusion in the hydrodynamic (velocity) and concentration (species) boundary layers. As the Schmidt number increases the concentration decreases. This causes the concentration buoyancy effects to be reduced which in turn decelerate the primary and secondary flows. Primary and secondary momentum boundary layer thickness will therefore be increased whereas species concentration boundary layer thickness will be enhanced with increasing Schmidt number. In all the computations Schmidt number is considered to be in excess of unity which are appropriate for transport phenomena in seawater MHD generators. Smaller values of $S c$ are equivalent to increasing chemical molecular diffusivity. Sc also represents the relative thickness of the velocity (hydrodynamic) boundary layer to the concentration (solutal) boundary layer. Larger $S c$ fluids have lower mass diffusion characteristics. For $S c$ greater than or equal to two (as studied in Figs. 19 and 20) the momentum diffusivity is twice the species diffusivity.

Figures 21 and 22 visualize the impact of the Soret (thermo-diffusion) number ( $S r$ ) on the velocity and concentration profiles are plotted in respectively. The Soret number $\mathrm{Sr}$ defines the effect of the temperature gradients inducing significant mass diffusion effects and is featured in the second order linear derivative term in Eqn. (15), viz $\operatorname{Sr} \partial^{2} \theta / \partial y^{2}$. Increasing Soret number $\mathrm{Sr}$ clearly elevates both the primary and secondary velocity magnitudes and furthermore also boosts the species concentration magnitudes. Both momenta boundary layer thicknesses are decreased whereas the species boundary layer thickness is enhanced. Inclusion of both Dufour and Soret cross-diffusion effects therefore exert a non-trivial influence on transport characteristics along the duct wall (plate). Absence of these effects results in a substantial under-prediction in primary and secondary velocity (without Dufour effect), under-prediction in species concentration (without Soret effect) and over-prediction in primary and secondary velocity magnitudes (without Soret effect). 
Figures 23-25 present the response in primary and secondary velocity components, temperature and concentration to variation in time $(t)$. In all these figures there is a significant elevation in velocities, temperature and species concentration with time elapse. In other words, these quantities are minimized at the initiation of flow and with progression in time they are substantially boosted. The modifications are very distinct and further emphasize the crucial need to include time-dependent effects in realistic generator MHD near wall flow dynamic simulations. Steady-state models are therefore liable to massively under-predict magnitudes of velocity components, temperature and concentration. This in turn would result in under-predictions of wall skin friction and over-prediction of wall heat transfer gradient (Nusselt number) and wall species gradient (Sherwood number) which would furnish inaccurate estimates of momentum, thermal and concentration boundary layer thicknesses for MHD generator design leading to possible complications and reduced efficiencies during operations, as noted by Cramer and Pai [60].

Figure 26 illustrates the influence of wall duct inclination angle $(\alpha)$ on primary and secondary velocity components. It is observed that an increase in inclination (increasingly sloped duct walls) evidently depresses both primary and secondary velocity magnitudes. Primary velocity overshoots are also markedly pushed closer to the wall and reduced simultaneously. At very high inclination (>35 degrees) primary velocity overshoots are eliminated. The inclination effect is simulated via the scaled buoyancy terms i.e. thermal buoyancy force term $(+G r \cos \alpha . \theta)$ and species buoyancy force $(+G m \cos \alpha$.C) which appear in the primary momentum Eqn. (12) only. With increasing angle of inclination $(\alpha)$ the buoyancy forces are decreased. The maximum buoyancy forces correspond to $\alpha=0$ i.e. vertical duct wall for which $\operatorname{Cos} \alpha \rightarrow 1$, the forces become $+\operatorname{Gr} \theta$ and species buoyancy force $+G m C$. As $\alpha$ increases, Cos $\alpha$ decreases and therefore both buoyancy forces are depleted. This inevitably opposes momentum development and results in deceleration in both the primary and secondary flow. The advantage however is that with inclined walls the flow can be regulated (similarly to with porous media) since the dual velocity fields are damped strongly. This is of potential benefit to MHD seawater generator designers. In conjunction with the time effect, the present model can therefore provide a wider range of design parameters for optimization of MHD generator performance and extension of, for example, cathode lifetime (lifetimes are dependent upon gap voltage and average duct wall metal temperature) and mitigation of chemical corrosion may be obtained with judicious combinations of the control parameters (magnetic field, Hall parameter, inclination, time, 
buoyancy effects, cross-diffusion effects and also with appropriate selection of the magnetic pumping fluid).

\section{CONCLUSIONS}

A mathematical model has been developed for time-dependent radiative magnetohydrodynamic flow, heat and mass transfer from an inclined wall embedded in a homogenous, isotropic high-permeability porous medium with chemical reaction, viscous dissipation and Hall current effects. The objective has been to evaluate in more detail the multichemo-physics of near wall duct boundary layer transport phenomena in real working Hall MHD energy generators which may experience corrosive, high temperature working conditions. The governing non-linear partial differential equations for mass, momentum, energy and species conservation have been converted into a system of coupled non-linear dimensionless partial differential equations with appropriate similarity variables and dimensionless numbers. The normalized conservation equations have been solved with a robust finite element method (MATLAB-FEM) subject to corresponding initial and boundary conditions. Detailed grid independence tests are conducted. Validation of solutions with published literature is also included for several special cases, namely non-reactive, nondissipative flow in the absence of heat generation or absorption. Further validation is included using a multi-step differential transform method (MS-DTM). The current computations have shown that:

(I) Primary velocity is enhanced with Eckert number, thermal Grashof number, solutal Grashof number, Hall parameter, permeability parameter, Dufour number, Soret number, radiation-conduction parameter and time whereas it is reduced with first order chemical reaction parameter, heat absorption, magnetic body force parameter, Prandtl number, Schmidt number and wall inclination.

(II) Secondary velocity is elevated with Eckert number, solutal Grashof number, thermal Grashof number, magnetic body force parameter, Hall parameter, radiationconduction parameter, Dufour number, Soret number and time whereas it is suppressed with reaction parameter, heat absorption, Prandtl number, Schmidt number and wall inclination. 
(III) Temperature is enhanced with Eckert number, Dufour number, heat absorption (sink), radiation-conduction parameter and time whereas it is depressed with Prandtl number.

(IV) Species concentration is reduced with increasing chemical reaction parameter (destructive homogenous reaction) and Schmidt number whereas it is elevated with Soret number and time.

(V) Primary and secondary skin-friction coefficients increase with an elevation in Dufour number, Hall parameter and Soret number whereas they are suppressed with a rise in magnetic body force parameter and wall inclination.

(VI) Primary and secondary skin-friction coefficients also are suppressed at the inclined duct wall with a decrease in permeability parameter and an increase in Schmidt number.

(VII) Nusselt number increases whereas the Sherwood number decreases with elevation in Dufour number and Soret number

(VIII) Nusselt number decreases whereas the Sherwood number increases with an enhancement in Schmidt number.

The present simulations provide an interesting insight into complex fluid/thermal/species diffusion characteristics in the boundary layer region of relevance to working MHD generator systems. Both MATLAB-FEM and MS-DTM numerical algorithms very successfully and efficiently solve multi-physical boundary value problems associated with such systems. However only homogenous reactions have been considered. Future studies will address more complex heterogeneous chemical reactions which also arise in high-temperature corrosion environments and will be communicated imminently.

\section{REFERENCES}

[1] H. K. Messerle, Magnetohydrodynamic Power Generation, John Wiley, New York (1994).

[2] L. Z. Zhao et al., Numerical analysis on a helical-channel seawater MHD generator, IEEE PES Innovative Smart Grid Technologies, 1-5 (2012).

[3] T. Okuo et al., Heavy metal electrodes development for coal-fired MHD generator channels, IEEE Trans. Plasma Science, 10, 115 (1990). 
[4] V.S. Slavin et al., Space power installation based on solar radiation collector and MHD generator, IEEE Transactions on Energy Conversion, 21, 491-503 (2006).

[5] A. Solbes et al., Design of a ten megawatt rocket driven disk MHD generator. Proceedings of the 24th Intersociety Energy Conversion Engineering Conference, Washington, DC, USA, USA, 6-11 Aug (1989).

[6] Y. Inui et al., Study of high-performance non-equilibrium MHD generator for compact fusion advanced Rankine cycle, Fusion Engineering and Design, 18, 233-238 (1991).

[7] A. A. Bednarczyk, Nuclear electric magnetohydrodynamic propulsion for submarines, MSc Thesis, Naval Architecture and Marine Engineering, Massachusetts Institute of Technology (MIT), USA, October (1989).

[8] D. L. Mitchell and D. U. Gubser, Magnetohydrodynamic ship propulsion with superconducting magnets, Journal of Superconductivity, 1, 349-364 (1988).

[9] L.Z. Zhao et al., MHD wave energy underwater recharging platforms for AUVs, $26^{T H}$ International Ocean and Polar Engineering Conference, Rhodes, Greece, June 26-July 1 (2016).

[10] G. T. Hummert, An evaluation of direct current electromagnetic propulsion in seawater, Office of Naval Research, Report ONR-CR168-007-1, Maryland, USA (1979).

[11] A. Arikoglu, Effect of slip on entropy generation in a single rotating disk in MHD flow, Applied Energy, 85, 1225-1236 (2008).

[12] M. Ishikawa, Stability analysis of MHD disk generators and application to power systems with CO2 recovery, Energy, 22, 239-247 (1997).

[13] L.L. Lengyel, Two-dimensional current distributions in Faraday type MHD energy converters operating in the nonequilibrium conduction mode, Energy Conversion, 9, 13-23 (1969).

[14] N. Stankiewicx and R. W. Palmer, Three-dimensional potential and current distributions in a Hall generator with assumed velocity profiles, NASA Lewis Research Centre, TN D-6622, January (1972).

[15] T. Hardianto, Three-dimensional flow analysis in a Faraday-type MHD generator, IEEE Transactions on Industry Applications, 44, 1116-1123 (2008).

[16] C. Das et al,, Some practical applications of magnetohydrodynamic pumping, Sensors and Actuators A: Physical, 201, 43-48 (2013). 
[17] A. Liberati and Y. Okuno, Influence of anode-region boundary-layer separation on disk MHD-generator performance, IEEE Transactions on Plasma Science, 35, 1588-1597 (2007).

[18] J. K. Koester and R. A. Perkins, Discharge and corrosion characteristics of slagging metal electrodes for MHD power generators, Journal of Materials for Energy Systems, 1, 41-54 (1979).

[19] J. Dong-Laing et al., A composite electrode material study and its performance in a MHD test unit, Seventh International Conference on MHD Electrical Power Generation, Massachusetts Institute of Technology, Cambridge, MA, June 16-20, pp. 292-299 (1980).

[20] J.B. Heywood and G.J. Womack, Open-Cycle MHD Power Generation, Pergamon Press, New York (1969).

[21] S. Jena, S. R. Mishra and G. C. Dash, Chemical reaction effect on MHD Jeffery fluid flow over a stretching sheet through porous media with heat generation/absorption. International Journal of Applied and Computational Mathematics. 3: 1225-1238 (2017).

[22] D. Srinivasacharya and G. Swamy Reddy, Chemical reaction and radiation effects on mixed convection heat and mass transfer over a vertical plate in power-law fluid saturated porous medium, Journal of the Egyptian Mathematical Society, 24: 108-115 (2016).

[23] O. Anwar Bég, M. Ferdows, Tasveer A. Bég, T. Ahmed, M. Wahiduzzaman, Md. M. Alam, Radiative optically-dense magnetized transient reactive transport phenomena with cross diffusion and dissipation effects: numerical simulations, J. Taiwan Inst. Chemical Engineers, 66, 12-26 (2016).

[24] W. C. Kennedy, W. F. Hughes, The steady state performance, magneto-acoustical response, and stability of flow in a Hall MHD generator, International Journal of Engineering Science, 11, 1143-1160 (1973).

[25] K. N. Venkatasiva Murthy, Hall effects on non-linear Hartmann-Ekman layers, International Journal of Engineering_Science, 21, 841-845 (1983).

[26] E. Sawaya, N. Ghaddar, F. Chaaban, Evaluation of the Hall parameter of electrolyte solutions in thermo-syphonic MHD flow, International Journal of Engineering Science, 40, 2041-2056 (2002).

[27] E. M. Aboeldahab and E. M. E. Elbarbary, Hall current effect on magnetohydrodynamic free-convection flow past a semi-infinite vertical plate with mass transfer, International Journal of Engineering Science, 39, 1641-1652 (2001). 
[28] O. Anwar Bég, J. Zueco and H.S. Takhar, Unsteady magnetohydrodynamic HartmannCouette flow and heat transfer in a Darcian channel with Hall current, ionslip, viscous and Joule heating effects: Network numerical solutions, Communications in Nonlinear Science Numerical Simulation, 14, 1082-1097 (2009).

[29] E. M. Abo-Eldahab and M. A. El Aziz, Viscous dissipation and Joule heating effects on MHD-free convection from a vertical plate with power-law variation in surface temperature in the presence of Hall and ion-slip currents, Applied Mathematical Modelling, 29, 579-595 (2005).

[30] A.M. Salem and M. A. El Aziz, Effect of Hall currents and chemical reaction on hydromagnetic flow of a stretching vertical surface with internal heat generation/absorption, Applied Mathematical Modelling, 32, 1236-1254 (2008).

[31] O. Anwar Bég, Lik Sim, J. Zueco and R. Bhargava, Numerical study of magnetohydrodynamic viscous plasma flow in rotating porous media with Hall currents and inclined magnetic field influence, Communications in Nonlinear Science and Numerical Simulation, 15, 345-359 (2010).

[32] Aurangzaib et al., Effect of thermal stratification on MHD free convection with heat and mass transfer over an unsteady stretching surface with heat source, Hall current and chemical reaction, International Journal of Advances in Engineering Sciences and Applied Mathematics, 4,_217-225 (2012).

[33] O. Anwar Bég, O., Rawat, S., Zueco, J., Osmond, L., R.S.R.Gorla. Finite element and network electrical simulation of rotating magnetofluid flow in nonlinear porous media with inclined magnetic field and Hall currents, Theor. Appl. Mech. 41:1-35 (2014).

[34] S. Das, B. C. Sarkar and R. N. Jana, Hall effect on MHD free convection boundary layer flow past a vertical flat plate. Meccanica. 48: 1387-1398 (2013).

[35] O. Anwar Bég, S. Abdul Gaffar, V. Ramachandra Prasad and M.J. Uddin, Computational solutions for non-isothermal, nonlinear magnetoconvection in porous media with Hall/ionslip currents and Ohmic dissipation, Engineering Science and Technology-International Journal, 19, 377-394 (2016).

[36] G.S. Seth,J.K. Singh, Mixed convection hydromagnetic flow in a rotating channel with Hall and wall conductance effects, Applied Mathematical Modelling, 40, 2783-2803 (2016).

[37] M. Kinyanjui, J. K. Kwanza, and S. M. Uppal, Magnetohydrodynamic free convection heat and mass transfer of a heat generating fluid past an impulsively started infinite vertical porous plate with Hall current and radiation absorption, Energy Conversion and Management, 42, 917-931 (2001).

[38] N.J.S.Maken and B. Gupta, Ionization instability in nonequilibrium MHD generators: Effect of diffusion, Energy Conversion and Management, 21, 115-120 (1981). 
[39] M.-C. Li, Y.-W. Tian, and Y.-C. Zhai, Soret and Dufour effects in strongly endothermic chemical reaction system of porous media, Transactions of Nonferrous Metals Society of China, 16, 1200-1204 (2006).

[40] O. Anwar Bég, Tasveer A. Bég, A.Y. Bakier, V. Prasad, Chemically-reacting mixed convective heat and mass transfer along inclined and vertical plates with Soret and Dufour effects: Numerical solutions, Int. J. Applied Mathematics and Mechanics, 5, 2, 39-57 (2009).

[41] S. N. Gaikwad, M. S. Malashetty, and K. Rama Prasad, An analytical study of linear and non-linear double diffusive convection with Soret and Dufour effects in couple stress fluid, International Journal of Non-Linear Mechanics, 42, 903-913 (2007).

[42] O. Anwar Bég, Bakier, A.Y. and V.R. Prasad, Numerical study of free convection magnetohydrodynamic heat and mass transfer from a stretching surface to a saturated porous medium with Soret and Dufour effects, Computational Materials Science, 46, 1, 57-65 (2009).

[43] M. Abd El-Aziz, Thermal-diffusion and diffusion-thermo effects on combined heat and mass transfer by hydromagnetic three-dimensional free convection over a permeable stretching surface with radiation, Physics Letters, Section A, 372, 263-272 (2008).

[44] O. Anwar Bég, V. R. Prasad, B. Vasu, N. Bhaskar Reddy, Q. Li and R. Bhargava, Free convection heat and mass transfer from an isothermal sphere to a micropolar regime with Soret/Dufour effects, Int. J Heat and Mass Transfer, 54, 9-18 (2011).

[45] Siva Reddy Sheri and R. Srinivasa Raju, Soret effect on unsteady MHD free convective flow past a semi-infinite vertical plate in the presence viscous dissipation, International Journal Computational Methods in Engineering Science and Mechanics.16: 132-141 (2015).

[46] N. Pandya and A. K. Shukla, Soret-Dufour and Radiation and hall effects on unsteady MHD flow of a viscous incompressible fluid past an inclined plate embedded in porous medium, TWMS J. App. Eng. Math. 6: 163-174 (2016).

[47] Siva Reddy Sheri and Prasanthi Modugula, Heat and mass transfer effects on unsteady MHD flow over an inclined porous plate embedded in porous medium with Soret-Dufour and chemical reaction. International Journal of Applied and Computational Mathematics. 3:12891306 (2017).

[48] A. Majeed et al., Chemical reaction and heat transfer on boundary layer Maxwell Ferrofluid flow under magnetic dipole with Soret and suction effects, Engineering Science and Technology- International Journal, 20, 1122-1128 (2017).

[49] O. Anwar Bég, S.K. Ghosh, M. Narahari and T. A. Bég, Mathematical modelling of thermal radiation effects on transient gravity-driven optically-thick gray convection flow along an inclined plate with pressure gradient, Chemical Engineering Communications, 198, 1-15 (2011).

[50] M.A.A. Mahmoud, Thermal radiation effects on MHD flow of a micropolar fluid over a stretching surface with variable thermal conductivity, Physical A, 375, 401-410 (2007). 
[51] O. Anwar Bég, J. Zueco and L. M. López-Ochoa, Network numerical analysis of opticallythick hydromagnetic slip flow from a porous spinning disk with radiation flux, variable thermophysical properties and surface injection effects, Chemical Engineering Communications, 198, 3, 360-384 (2011).

[52] El-Hakiem M. A. MHD oscillatory flow on free convection radiation through a porous medium with constant suction velocity. J Magnetism and Magnetic Materials, 220, 271-276. (2000).

[53] Shamshuddin MD, Siva Reddy Sheri and O Anwar Bég, Oscillatory dissipative conjugate heat and mass transfer in chemically reacting micropolar flow with wall couple stress: A finite element numerical study, Proc IMechE Part E: J Process Mechanical Engineering (2017). DOI: $10.1177 / 0954408917743372$ (17 pages)

[54] O. Anwar Bég, J. Zueco, S. K. Ghosh and Alireza Heidari, Unsteady magnetohydrodynamic heat transfer in a semi-infinite porous medium with thermal radiation flux: analytical and numerical study, Advances in Numerical Analysis, 2011, 1-17 (2011). Article ID 304124, doi: 10.1155/ 2011/ 304124 .

[55] F.M. Hardy, F.S. Ibrahim, S.M. Abel Gaiedz, M.R. Eid, Radiation effect on viscous flow of a nanofluid and heat transfer over a nonlinearly stretching sheet, Nanoscale Res Lett., 7. 113 (2012).

[56] M. M. Bhatti, A. Zeeshan, N. Ijaz, O. Anwar Bég and A. Kadir, Mathematical modelling of nonlinear thermal radiation effects on EMHD peristaltic pumping of viscoelastic dusty fluid through a porous medium channel, Engineering Science and Technology (2016). (11 pages) http://dx.doi.org/10.1016/j.jestch.2016.11.003

[57] P. Mohankrishna, V. Sugunamma, N. Sandeep, Radiation and magnetic field effects on unsteady natural convection flow of a nanofluid past an infinite vertical plate with heat source, Chem Process Eng. Res, 25, 39-52 (2014).

[58] M.D. Shamshuddin, S.R. Mishra, O. Anwar Bég and A. Kadir, Unsteady reactive magnetic radiative micropolar flow, heat and mass transfer from an inclined plate with Joule heating: a model for magnetic polymer processing, Proc. IMechE- Part C. - Mechanical Engineering Science (2018). DOI: 10.1177/0954406218768837 (16 pages)

[59] M.A. Seddeek, AA Darwish and MS Abdelmeguid, Effects of chemical reaction and variable viscosity on hydromagnetic mixed convection heat and mass transfer for Hiemenz flow through porous media with radiation, Communications in Nonlinear Science and Numerical Simulation, 12 (2) 195-213 (2007).

[60] K.C. Cramer and S.I. Pai, Applied Magnetofluid Dynamics for Engineers and Applied Physicists, MacGraw-Hill, New York (1973).

[61] O. Anwar Bég, Numerical methods for multi-physical magnetohydrodynamics, Chapter 1, pp. 1-112, New Developments in Hydrodynamics Research, M. J. Ibragimov and M. A. Anisimov, Eds., Nova Science, New York, September (2012). 
[62] J.N. Reddy, An Introduction to the Finite Element Method. McGraw-Hill Book Company. New York, USA (2006).

[63] J. K. Zhou, Differential Transformation and Applications for Electrical Circuits, Huazhong University of Science \& Technology Press, Wuhan, China (1986).

[64] O. Anwar Bég, M.M. Rashidi, and N. Freidooni Mehr, Second law analysis of hydromagnetic flow from a stretching rotating disk: DTM-Padé simulation of novel nuclear MHD propulsion systems, Frontiers of Aerospace Engineering, 2, 1, 29-38 (2013).

[65] R.J. Pollina, W. Simpsom and L.C. Farrar, MHD channel gas-side element erosioncorrosion studies, Symposium on Engineering Aspects of Magnetohydrodynamics, University of Chicago, Illinois, USA, 26-28 Jun (1990).

[66] Ali Kadir, O. Anwar Bég, Mohammed El Gendy, Tasveer A. Bég and M.D. Shamshuddin, Computational fluid dynamic and thermal stress analysis of coatings for high-temperature corrosion protection of aerospace gas turbine blades, Comp. Thermal Sci. (2018) Under Review

[67] R. J. Rosa. Magnetohydrodynamic Energy Conversion, Washington, Hemisphere Pub. Corp, USA (1987).

[68] W. Ibrahim and B. Shankar, MHD boundary layer flow and heat transfer of a nanofluid past a permeable stretching sheet with velocity, thermal and solutal slip boundary conditions. Comput. Fluids, 75:1-10 (2013). 